\title{
Pharmacotherapy for Cocaine Use Disorder-a Systematic Review and Meta-analysis
}

\author{
Brian Chan, $M D M P H^{1,2}$, Karli Kondo, PhD MA ${ }^{3,4}$, Michele Freeman, $\mathrm{MPH}^{3}$, \\ Chelsea Ayers, $B A^{3}$, Jessica Montgomery, $\mathrm{MPH}^{3}$, and Devan Kansagara, MD MCR ${ }^{1,3,5}$
}

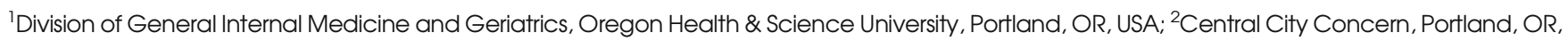
USA; ${ }^{3}$ Evidence Synthesis Program Center, VA Portland Health Care System, Portland, OR, USA; ${ }^{4}$ Research Integrity Office, Oregon Health \& Science University, Portland, OR, USA; ${ }^{5}$ Department of Medicine, VA Portland Health Care System, Portland, OR, USA.

BACKGROUND: Currently, there are no accepted FDAapproved pharmacotherapies for cocaine use disorder, though numerous medications have been tested in clinical trials. We conducted a systematic review and metaanalysis to better understand the effectiveness of pharmacotherapy for cocaine use disorder.

METHODS: We searched multiple data sources (MEDLINE, PsycINFO, and Cochrane Library) through November 2017 for systematic reviews and randomized controlled trials (RCTs) of pharmacological interventions in adults with cocaine use disorder. When possible, we combined the findings of trials with comparable interventions and outcome measures in random-effects metaanalyses. We assessed the risk of bias of individual trials and the strength of evidence for each outcome using standardized criteria. Outcomes included continuous abstinence (3+ consecutive weeks); cocaine use; harms; and study retention. For relapse prevention studies (participants abstinent at baseline), we examined lapse (first cocaine positive or missing UDS) and relapse (two consecutive cocaine positive or missed UDS').

RESULTS: Sixty-six different drugs or drug combinations were studied in seven systematic reviews and 48 RCTs that met inclusion criteria. Antidepressants were the most widely studied drug class (38 RCTs) but appear to have no effect on cocaine use or treatment retention. Increased abstinence was found with bupropion (2 RCTs: RR 1.63, 95\% CI 1.02 to 2.59), topiramate (2 RCTs: RR 2.56, 95\% CI 1.39 to 4.73 ), and psychostimulants (14 RCTs: RR 1.36, 95\% CI 1.05 to 1.77 ), though the strength of evidence for these findings was low. We found moderate strength of evidence that antipsychotics improved treatment retention (8 RCTs: RR 1.33, 95\% CI 1.03 to 1.75).

DISCUSSION: Most of the pharmacotherapies studied were not effective for treating cocaine use disorder.

\footnotetext{
Prior Presentations

This paper was accepted, but not presented at the annual meeting of the Society of Behavioral Medicine (March 2019). It has also been accepted to the annual meeting of the Society of General Internal Medicine (Poster; May 2019), College on Problems of Drug Dependence (Poster; June 2019), and the American Psychological Association's annual convention (Poster and "Rapid Response"; August 2019).
}

Electronic supplementary material The online version of this article (https://doi.org/10.1007/s11606-019-05074-8) contains supplementary material, which is available to authorized users.

Received April 29, 2019

Accepted May 1, 2019

Published online June 10, 2019
Bupropion, psychostimulants, and topiramate may improve abstinence, and antipsychotics may improve retention. Contingency management and behavioral interventions along with pharmacotherapy should continue to be explored.

\section{SR REGISTRATION: Prospero CRD42018085667}

KEY WORDS: substance use; pharmacotherapy; systematic review; cocaine.

$\mathrm{J}$ Gen Intern Med 34(12):2858-73

DOI: $10.1007 / \mathrm{s} 11606-019-05074-8$

(C) Society of General Internal Medicine (This is a U.S. government work and not under copyright protection in the U.S.; foreign copyright protection may apply) 2019

\section{INTRODUCTION}

Cocaine use disorder remains a serious problem in the USA and worldwide. In the USA, 900,000 adults met criteria for cocaine use disorder in 2014 and $40 \%$ of visits to emergency departments for drug misuse or abuse involved cocaine. ${ }^{1}$ Cocaine use is associated with cardiovascular and neurologic effects, and chronic repeated exposure leads to tolerance, adverse psychological and behavioral effects, and complications including infections, stroke, and seizure. ${ }^{2,3}$

Psychosocial and behavioral therapies, including cognitive behavioral therapy (CBT) and contingency management (CM) interventions, are the primary treatments for cocaine use disorder. However, they are time-consuming, not universally accessible, and suffer from low treatment retention. Currently, there are no Food and Drug Administration (FDA)-approved medications to treat cocaine use disorder. One challenge in establishing the evidence base for pharmacotherapy of cocaine use disorder is the sheer number of drug classes that have been studied. Prior systematic reviews (SRs) have largely focused on single drugs ${ }^{4}$ or drug classes (anticonvulsants/ carbamazepine, ${ }^{5}$ dopamine agonists, ${ }^{6}$ psychostimulants, ${ }^{7}$ and antipsychotics $^{8}$ ). To our knowledge, none have examined the treatment of cocaine use disorder across drug classes. This SR examines the benefits and harms of pharmacological interventions for cocaine use disorder, and was part of a larger report of stimulant use disorders commissioned by the Veterans Health Administration (VHA). 


\section{METHODS}

\section{Data Sources and Search Strategies}

We searched MEDLINE, PsycINFO, and EBM Reviews Cochrane Database of Systematic Reviews through November 2017 (Online Appendix Table 1). We reviewed the bibliographies of relevant articles and contacted experts to identify additional studies. To identify in-progress or unpublished studies, we searched ClinicalTrials.gov, OpenTrials, and the World Health Organization (WHO) International Clinical Trials Registry Platform (ICTRP). The review protocol was registered to PROSPERO before we initiated the study (CRD42018085667). Our methods and reporting follow PRISMA guidelines. ${ }^{9}$

\section{Study Selection}

Study selection was guided by an analytic framework (Online Appendix Figure 1). We included randomized controlled trials (RCTs) of adults with cocaine use disorder that compared pharmacotherapies (head to head), to placebo, usual care, or psychotherapy. We also included RCTs that had been included in existing good quality SRs. We excluded studies examining patients with comorbid psychotic spectrum or bipolar disorders. We excluded studies that did not perform urine drug screens (UDS) at least once per week. PICOTS and study selection criteria are specified in Online Appendix Tables 2 and 3.

We dual reviewed and evaluated titles and abstracts for $18.6 \%$ of the search yield to ensure reliability. Two investigators independently reviewed the full text of all potentially relevant articles for inclusion. All discordant results were resolved through consensus or consultation with a third reviewer.

\section{Data Abstraction and Quality Assessment}

One investigator abstracted, and a second investigator confirmed details related to study design, setting, population, intervention and follow-up, co-interventions, outcomes, and harms. Two reviewers independently assessed the quality of each RCT using a tool developed by the Cochrane Collaboration $^{10}$ (Online Appendix Table 8). We directly report the findings from previous SRs as well as their assessments of study quality.

Our outcomes of interest were sustained abstinence (three or more weeks of negative UDS'), ${ }^{11}$ cocaine use, treatment retention, serious adverse events (SAEs), and treatment dropouts due to adverse events (AEs). For relapse prevention studies of participants abstinent at baseline, we examined lapse (first cocaine positive or missed UDS) and relapse (two consecutive cocaine positive or missed UDS'). For outcomes related to abstinence and use, we excluded studies relying on selfreported drug use, with the exception of findings from previous SRs.

\section{Data Synthesis and Analysis}

We qualitatively synthesized the evidence and separately examined the findings in patients with comorbid opioid use disorder. When possible, we combined data from trials as they were reported in previous SRs with data we abstracted directly from newer RCTs identified in our search in random-effects meta-analyses. ${ }^{12}$ We used RevMan $5.3^{13}$ to calculate the overall relative risk (RR) and 95\% CI of each outcome in the active treatment group compared with placebo. We assessed statistical heterogeneity among the pooled studies using the $I^{2}$ statistic. $^{14,15}$

We assessed the overall strength of evidence (SOE) for each outcome using an established method, and classified SOE as high, moderate, low, or insufficient. ${ }^{16}$

\section{RESULTS}

Our larger search for stimulant use disorders yielded 5564 citations. After reviewing the full text of 354 studies, we included seven systematic reviews and 48 RCTs specific to cocaine use disorder (Fig. 1). The included SRs and RCTs examined 66 different drugs including antidepressants, antipsychotics, anxiolytics, cognitive enhancing drugs, dopamine agonists, muscle relaxants, anticonvulsants, medications approved by the FDA for other substance use disorders, and a wide range of other pharmacotherapies (Online Appendix Table 4).

Table 1 presents a brief summary of findings for all drug classes. Table 2 provides a more detailed summary of the evidence on all pharmacotherapies for cocaine use disorder, stratified by drug class. The characteristics and findings of individual studies are provided in Online Appendix Tables 5 and 6.

\section{Psychopharmacotherapies}

Antidepressants: Bupropion, Desipramine, Fluoxetine, Mirtazapine, Nefazodone, Paroxetine, Sertraline, Venlafaxine. Antidepressants were the most widely studied among the drug classes. We found 34 trials from two previous systematic reviews ${ }^{7,17}$ and four subsequent trials ${ }^{18-21}$ investigating antidepressants (including bupropion) for cocaine use disorder. The more recent trials examine sertraline, ${ }^{20,21}$ venlafaxine, ${ }^{18}$ and mirtazapine. ${ }^{19}$ Overall, there were no differences on sustained abstinence, use, retention, or harms outcomes.

In a meta-analysis combining $10 \mathrm{RCTs}^{7,}, 17,18$ across all antidepressants, abstinence occurred more frequently in the antidepressant groups than placebo (RR 1.27, 95\% CI 0.99 to 1.63), but the difference did not reach statistical significance $(P=0.06$; Online Appendix Figure 2). We found moderate SOE of no difference on cocaine use between antidepressants as a class and placebo. Findings were consistent across four RCTs reported in a systematic review ${ }^{17}$ (RR $1.05,95 \%$ CI 0.91 to 1.21 ) and two additional RCTs. ${ }^{18,}{ }^{19}$ We found high 
5,564 Citations identified from electronic database searches:

4,396 from PubMed/Ovid MEDLINE (October, 2017)

624 from PsycINFO (November, 2017)

467 from Ovid EBM Reviews (CDSR, DARE, HTA, Cochrane CENTRAL; November, 2017

77 from WHO ICTRP (November 2017)

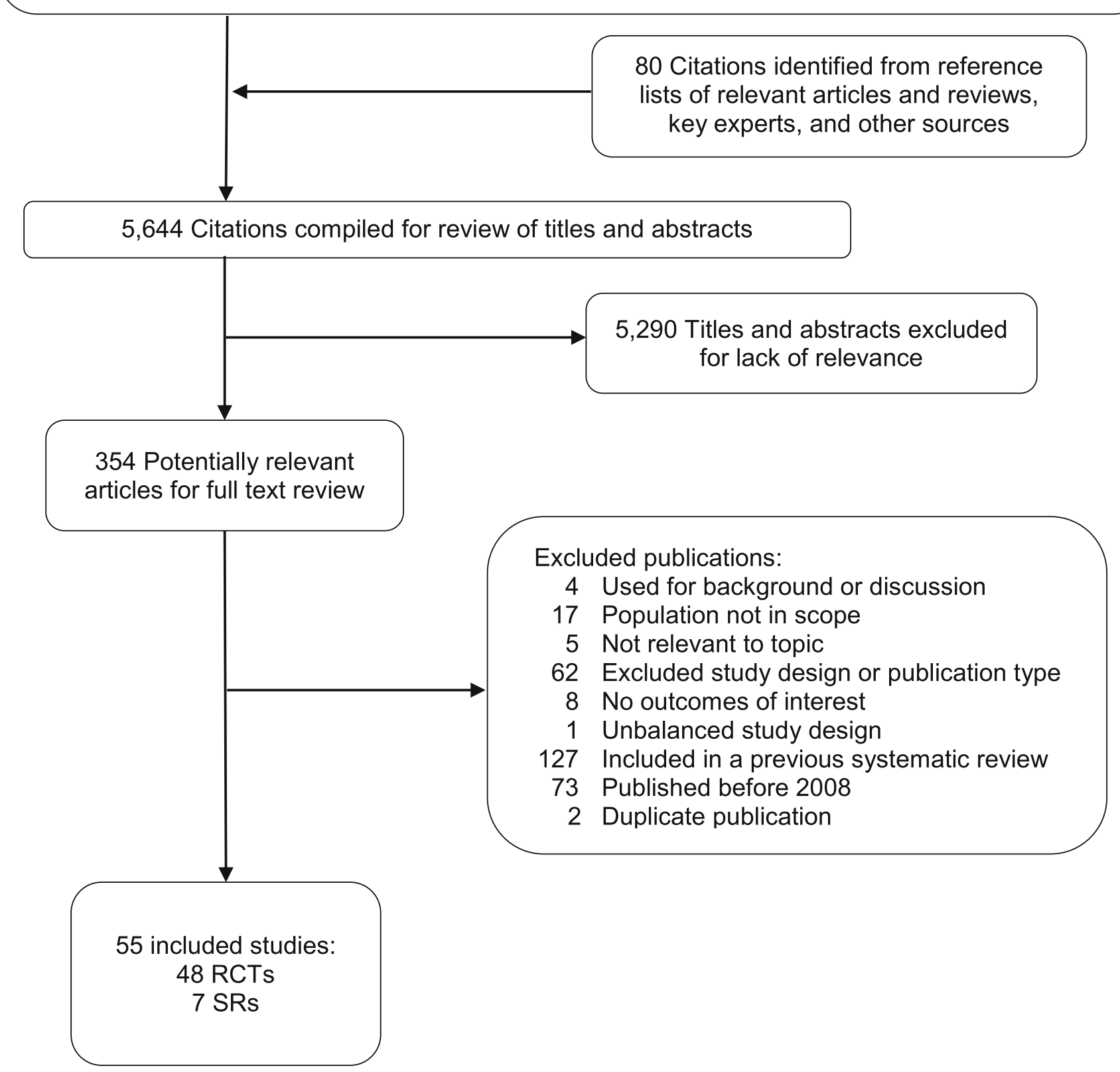

Figure 1 Literature flow diagram. RCT, randomized controlled trial; SR, systematic review.

SOE that antidepressants as a class are no better than placebo for study retention (33 RCTs; RR $0.95,95 \%$ CI 0.87 to 1.03 ; Fig. 2). There were no differences in dropouts due to AEs (moderate SOE) or SAEs (low SOE).

Selective Serotonin Reuptake Inhibitors: Fluoxetine, Paroxetine, and Sertraline. We found two low risk of bias (ROB) $\mathrm{RCTs}^{20,21}$ and seven RCTs in the SR of antidepressants ${ }^{17}$ that provide moderate SOE that selective serotonin reuptake inhibitors (SSRIs) do not improve study retention $(N=527$; RR $0.94,95 \%$ CI 0.68 to 1.29 ; Fig. 2). However, three RCTs $(N=251)$ in the $\mathrm{SR}^{17}$ provide low-strength evidence of a higher risk of dropout due to AEs with SSRIs compared with placebo (RR 3.55, 95\% CI 1.11 to 11.34 ). 
Table 1 Brief Summary of Findings

\begin{tabular}{|c|c|c|c|c|c|c|}
\hline & Abstinence & Use & Lapse & Relapse & Retention & Harms \\
\hline $\begin{array}{l}\text { All Antidepressants: Bupropion, } \\
\text { Desipramine, Fluoxetine, } \\
\text { Mirtazapine, Nefazodone, } \\
\text { Paroxetine, Sertraline, Venlafaxine }\end{array}$ & $\star \star$ & $\star \star$ & $\star$ & $\star \star$ & $\star \star \star$ & $\star \star$ \\
\hline Aminoketone: Bupropion & $\star$ & $\star$ & NA & NA & $\star \star$ & $\varnothing$ \\
\hline $\begin{array}{l}\text { SSRIs: Fluoxetine, } \\
\text { Paroxetine, and Sertraline }\end{array}$ & NA & NA & $\varnothing$ & $\varnothing$ & $\star \star$ & $\star$ \\
\hline $\begin{array}{l}\text { SSRI in patients abstinent at } \\
\text { Baseline: Sertraline }\end{array}$ & NA & NA & $\star$ & $\star$ & $\star$ & $\varnothing$ \\
\hline $\begin{array}{l}\text { All Antipsychotics: Aripiprazole, } \\
\text { Haloperidol, Lamotrigine, } \\
\text { Olanzapine, Quetiapine, } \\
\text { Risperidone, Reserpine }\end{array}$ & $\star$ & $\star$ & $\varnothing$ & $\varnothing$ & $\star \star$ & $\varnothing$ \\
\hline $\begin{array}{l}\text { Psychostimulants: } \\
\text { Dexamphetamine, } \\
\text { Lisdexamfetamine, Mazindol, } \\
\text { Methamphetamine, } \\
\text { Methylphenidate, Mixed } \\
\text { Amphetamine Salts, Modafinil, } \\
\text { Selegiline }\end{array}$ & $\star$ & $\star$ & NA & NA & $\star \star$ & $\star \star$ \\
\hline $\begin{array}{l}\text { Cognitive Enhancing Drugs: } \\
\text { Memantine, Atomoxetine }\end{array}$ & $\varnothing$ & $\varnothing$ & NA & $\varnothing$ & $\varnothing$ & $\varnothing$ \\
\hline Anxiolytic: Buspirone & $\varnothing$ & NA & $\varnothing$ & $\varnothing$ & $\varnothing$ & $\varnothing$ \\
\hline $\begin{array}{l}\text { Anticonvulsants/Muscle } \\
\text { Relaxants: Baclofen, } \\
\text { Carbamazepine, Gabapentin, } \\
\text { Lamotrigine, Phenytoin, Tiagabine, } \\
\text { Topiramate, Vigabatrin }\end{array}$ & NA & $\star \star$ & NA & NA & $\star \star$ & $\varnothing$ \\
\hline Anticonvulsant: Topiramate & $\star$ & $\varnothing$ & NA & NA & $\star \star$ & $\varnothing$ \\
\hline $\begin{array}{l}\text { Drugs for other substance use } \\
\text { disorders: Acamprosate, } \\
\text { Buprenorphine, Buprenorphine + } \\
\text { Naloxone, Disulfiram, Naltrexone, } \\
\text { Methadone, Varenicline }\end{array}$ & $\star$ & $\varnothing$ & $\varnothing$ & $\varnothing$ & $\varnothing$ & $\varnothing$ \\
\hline Disulfiram & $\star$ & $\star$ & NA & NA & $\star \star$ & $\star$ \\
\hline $\begin{array}{l}\text { Dopamine agonists: Amantadine, } \\
\text { bromocriptine, L dopa/Carbidopa, } \\
\text { pergolide, cabergoline hydergine, } \\
\text { and pramipexole }\end{array}$ & $\star$ & NA & NA & NA & $\star \star$ & NA \\
\hline
\end{tabular}

Shading represents the direction of effect:

$\begin{array}{cl}\text { (No color) } & \text { Unclear } \\ \text { Grey } & \text { No difference } \\ \text { Green } & \text { Evidence of benefit } \\ \text { Red } & \text { Favors placebo }\end{array}$

Relapse Prevention: Sertraline. We found two trials $(N=116)$ examining sertraline for relapse prevention among subjects who were cocaine-abstinent at baseline. ${ }^{20,} 21$ These RCTs provided a 2 -week residential treatment program during which subjects were required to achieve abstinence in order to continue treatment in a 10 -week outpatient program. Patients treated with sertraline were less likely to experience lapse (first cocaine positive or missing UDS samples [combined RR 0.80, 95\% CI 0.63 to 1.02]) and relapse (two consecutive cocaine positive or missing UDS [combined RR $0.75,95 \%$ CI 0.58 to 0.98; Online Appendix Figure 3]), although only the latter
Symbols represent the strength of the evidence:

NA No evidence or not applicable

$\varnothing \quad$ Insufficient

$\star \quad$ Low

$\star \star \quad$ Moderate

$\star \star \star \quad$ High

finding was statistically significant. Retention was also higher versus placebo (combined RR 1.43, 95\% CI 0.94 to 2.15; Online Appendix Figure 4), though the difference was not statistically significant $(P=0.09)$.

Bupropion. There were three trials reported in two existing $\mathrm{SRs}^{7,}{ }^{17}$ that examined bupropion for cocaine use disorder. There was low SOE that bupropion improved abstinence versus placebo ( $2 \mathrm{RCTs}$; combined RR 1.63, 95\% CI 1.03 to 2.59). ${ }^{7}$ Bupropion had no effect on cocaine use (low SOE) or retention (3 RCTs, combined RR 0.94, 95\% CI 0.76 to 1.15 ; 
Table 2 Summary of the Evidence on Pharmacotherapies for Cocaine Use Disorder, Stratified by Drug Class

\begin{tabular}{|c|c|c|c|c|}
\hline Outcomes & $\begin{array}{l}N \text { studies per outcome; } R O B \\
(N=\text { combined participants })\end{array}$ & Summary of findings by outcome & $\begin{array}{l}\text { Strength of } \\
\text { evidence* }\end{array}$ & $\begin{array}{l}\text { Comments and rationale for } \\
\text { strength of evidence rating }\end{array}$ \\
\hline \multicolumn{5}{|c|}{ Psychopharmacotherapies (antidepressants, antipsychotics, anxiolytics, cognitive enhancing drugs, and psychostimulants) } \\
\hline \multicolumn{5}{|c|}{ Äntidepressants (all) } \\
\hline & 1 low-ROB RCT ${ }^{18}(N=130)$ & $\begin{array}{l}10 \text { RCTs, } N=1226 \text {, } \\
\text { RR } 1.27(95 \% \text { CI } 0.99 \text { to } 1.63)\end{array}$ & Moderate & $\begin{array}{l}\text { benefit disappeared when restricted } \\
\text { co studies using strict criteria for } \\
\text { cocaine dependence. }\end{array}$ \\
\hline Use & $\begin{array}{l}1 \text { SR of } 4 \operatorname{RCTS}^{17}(N=251) \\
1 \text { low-ROB RCT }{ }^{18}(N=130) \\
1 \text { high-ROB RCT }{ }^{19}(N=24)\end{array}$ & $\begin{array}{l}\text { No difference. } 1 \text { SR reported a } \\
\text { combined use of cocaine (self- } \\
\text { reported or objective) RR of } 1.05 \\
\text { ( } 95 \% \text { CI } 0.91 \text { to } 1.21 \text { ). Similar } \\
\text { findings were reported in both } \\
\text { more recent low-ROB and high- } \\
\text { ROB RCTs. }\end{array}$ & Moderate & Indirectness (of outcome) \\
\hline Lapse & 2 low-ROB RCTs ${ }^{20,21}(N=116)$ & $\begin{array}{l}\text { Favors antidepressants. Participants } \\
\text { abstinent at baseline with } 1 \text { cocaine } \\
\text { positive UDS, combined RR } 0.79 \\
(95 \% \text { CI } 0.62 \text { to } 1.00 \text { ). }\end{array}$ & Low & $\begin{array}{l}\text { Small body of evidence } \\
\text { Indirectness (of results to general } \\
\text { population-participants had } \\
\text { achieved abstinence prior to the }\end{array}$ \\
\hline Relapse & & $\begin{array}{l}\text { Favors antidepressants. } \\
\text { Participants abstinent at baseline } \\
\text { with } 2 \text { consecutive cocaine } \\
\text { positive UDS', combined RR } 0.74 \\
(95 \% \text { CI } 0.57 \text { to } 0.96 \text { ). }\end{array}$ & Low & $\begin{array}{l}\text { outpatient phase). Lapse is defined } \\
\text { as the first cocaine positive or } \\
\text { missing UDS; relapse is } 2 \text { consec- } \\
\text { utive cocaine positive or missing } \\
\text { UDS'. }\end{array}$ \\
\hline Retention & $\begin{array}{l}1 \mathrm{SR} \text { of } 27 \mathrm{RCTs}^{17}(N=2417) \\
3 \text { low-ROB RCTs }{ }^{18,20,21}(N=263)\end{array}$ & $\begin{array}{l}\text { No difference. Meta-analysis of } \\
33 \text { RCTs } N=2918 \text {, RR } 0.95 \\
\text { ( } 95 \% \text { CI } 0.87 \text { to } 1.03 \text { ) }\end{array}$ & High & $\begin{array}{l}\text { Findings were similar in analyses } \\
\text { limited to RCTs specifying DSM } \\
\text { cocaine dependence criteria for } \\
\text { inclusion. }\end{array}$ \\
\hline Harms & $\begin{array}{l}1 \text { SR of } 13 \operatorname{RCTs}^{17}(N=1396) \\
1 \text { low-ROB RCT }{ }^{18}(N=130) \\
1 \text { high-ROB RCT }\end{array}$ & $\begin{array}{l}\text { No difference. } 1 \text { SR reported a } \\
\text { combined withdrawal due to an } \\
\text { adverse event RR of } 1.39 \text { ( } 95 \% \\
\text { CI } 0.91 \text { to } 2.12 \text { ). Two more recent } \\
\text { RCTs (1 low-ROB, } 1 \text { high-ROB) } \\
\text { reported consistent findings. } \\
\text { No difference. Two RCTs found } \\
\text { no difference in severe adverse }\end{array}$ & $\begin{array}{l}\text { Withdrawal } \\
\text { due to AEs: } \\
\text { Moderate } \\
\text { Severe AEs: } \\
\text { low }\end{array}$ & $\begin{array}{l}\text { Treatment withdrawal findings are } \\
\text { from } 1 \text { low and } 1 \text { high RCT and a } \\
\text { SR/meta-analysis of } 37 \text { RCTs. The } \\
\text { SR included studies with any defini- } \\
\text { tion of cocaine dependence or abuse. } \\
\text { Findings of SAEs are from a small } \\
\text { body of evidence. }\end{array}$ \\
\hline
\end{tabular}

Antidepressants (tricyclics)
Abstinence $\quad 1$ SR of $5 \operatorname{RCTs}^{17}(N=367)$

Use $\quad 1 \mathrm{SR}$ of $2 \mathrm{RCTs}^{17}(N=37)$

Retention $\quad 1 \mathrm{SR}$ of $15 \operatorname{RCTs}^{17}(N=1141)$

Harms $\quad 1 \mathrm{SR}$ of $5 \mathrm{RCTs}^{17}(N=381)$

Antidepressants (SSRIs): fluoxetine and sertraline

\begin{tabular}{|c|c|}
\hline Abstinence & NA \\
\hline & NA \\
\hline Relapse & 2 low-ROB RCTs ${ }^{20,21}(N=133)$ \\
\hline
\end{tabular}

Lapse

Retention $\quad 1 \mathrm{SR}$ of $7 \mathrm{RCTs}^{17}(N=527)$ 2 low-ROB RCTs ${ }^{20,21}(N=133)$ events by group.

No difference. $3+$ week abstinence, combined RR 1.55 (95\% CI 1.10 to 2.17$)$. Limited to DSM criteria for cocaine dependence (3 studies, $N=234)$ : combined RR 1.41 , (95\% CI 0.93 to 2.14 ).

No difference. Use of cocaine (self- Insufficient reported or objective), combined RR 0.85 (95\% CI 0.34 to 2.11)

No difference. Number of participants who did not complete the trial, combined RR 1.00 (95\% CI 0.85 to 1.18 )

No difference. Withdrawal due to an adverse event, combined RR 1.24 (95\% CI 0.64 to 2.43 ) SAE: NA

Low

NA

NA

Favors sertraline. Participants abstinent at baseline with 2 consecutive cocaine positive UDS', combined RR 0.74 (95\% CI 0.57 to 0.96 ).

Favors sertraline. Abstinent at baseline participants with 1 cocaine positive UDS, combined RR 0.79 (95\% CI 0.62 to 1.00 ). No difference. The SR's combined RR for participants not completing the trial was 0.99 (95\% CI 0.70 to 1.71). No difference in 2 more recent RCTs.

High

Moderate

Low
$4 / 5$ studies are of desipramine.

Small body of evidence. Imprecise estimate. Indirectness (of outcome)

Findings were similar in an analysis limited to RCTs specifying DSM cocaine dependence criteria for inclusion and in an analysis excluding high-ROB trials. 13/15 studies are of desipramine.

No evidence: Findings were similar in analyses limited to RCTs specifying DSM cocaine dependence criteria for inclusion. Imprecise estimate. $4 / 5$ studies are of desipramine.

No evidence NA

No evidence NA

Low $\quad$ Small body of evidence

Indirectness (of results to general population - participants had achieved abstinence prior to the outpatient phase). Lapse is defined as the first cocaine positive UDS, relapse is 2 consecutive cocaine positive UDS'.

Moderate Inconsistent results. Findings favored placebo when excluding 1 outlier, and no difference was found when further excluding 1 high-ROB RCT. Indirectness (of population)- 2 more 
Table 2. (continued)

\begin{tabular}{|c|c|c|c|c|}
\hline Outcomes & $\begin{array}{l}N \text { studies per outcome; ROB } \\
(N=\text { combined participants) }\end{array}$ & Summary of findings by outcome & $\begin{array}{l}\text { Strength of } \\
\text { evidence* }\end{array}$ & $\begin{array}{l}\text { Comments and rationale for } \\
\text { strength of evidence rating }\end{array}$ \\
\hline Harms & $1 \mathrm{SR}$ of $3 \mathrm{RCTs}^{17}(N=251)$ & $\begin{array}{l}\text { Favors placebo. Withdrawal due } \\
\text { to an adverse event, combined } \\
\text { RR 3.55 (95\% CI } 1.11 \text { to } 11.34) \text {. } \\
\text { SAE: NA }\end{array}$ & $\begin{array}{l}\text { Low } \\
\text { No evidence: } \\
\text { SAE }\end{array}$ & $\begin{array}{l}\text { recent RCTs enrolled only patients } \\
\text { who had achieved abstinence. } \\
\text { Imprecise estimate, small body of } \\
\text { evidence }\end{array}$ \\
\hline \multicolumn{5}{|c|}{ Antidepressant (SNRI): venlafaxine } \\
\hline Abstinence & 1 low-ROB RCT ${ }^{18}(N=130)$ & $\begin{array}{l}\text { No difference. } 1 \text { RCT found no } \\
\text { difference in } 3+\text { week abstinence } \\
\text { between groups }(P=0.94) \text {. }\end{array}$ & Insufficient & 1 single site study. \\
\hline Use & & $\begin{array}{l}\text { No difference. } 1 \text { RCT found no } \\
\text { difference in negative UDS' } \\
\text { between groups }(P=0.74) \text {. }\end{array}$ & Insufficient & \\
\hline Retention & & $\begin{array}{l}\text { No difference. } 1 \text { RCT found no } \\
\text { difference in retention between } \\
\text { groups. }\end{array}$ & Insufficient & \\
\hline Harms & & $\begin{array}{l}\text { No difference. } 1 \text { RCT found no } \\
\text { difference in withdrawals due to } \\
\text { adverse events by group. } \\
\text { No difference. } 1 \text { RCT found no } \\
\text { difference in severe AEs between } \\
\text { groups. }\end{array}$ & Insufficient & \\
\hline \multicolumn{5}{|c|}{ Antidepressant (Atypical): mirtazapine } \\
\hline Abstinence & & NA & No evidence & NA \\
\hline Use & 1 high-ROB $\mathrm{RCT}^{19}(N=24)$ & $\begin{array}{l}\text { No difference. } 1 \text { RCT found no } \\
\text { difference in study period use } \\
\text { between groups. }\end{array}$ & Insufficient & $\begin{array}{l}1 \text { very small underpowered study. } \\
\text { Details regarding randomization } \\
\text { and allocation concealment NR. }\end{array}$ \\
\hline Retention & NA & NA & No evidence & NA \\
\hline Harms & 1 high-ROB RCT ${ }^{19}(N=24)$ & $\begin{array}{l}\text { No difference. } 1 \text { RCT found no } \\
\text { difference in withdrawals due to } \\
\text { AEs between groups (none). } \\
\text { No difference. } 1 \text { RCT found no } \\
\text { difference in severe AEs between } \\
\text { groups (because there were none). }\end{array}$ & Insufficient & $\begin{array}{l}1 \text { very small underpowered study. } \\
\text { Details regarding randomization } \\
\text { and allocation concealment NR. }\end{array}$ \\
\hline \multicolumn{5}{|c|}{ Antidepressant (aminoketone): bupropion } \\
\hline Abstinence & $1 \mathrm{SR}^{2} 2 \mathrm{RCTs}^{7}(N=176)$ & $\begin{array}{l}\text { Favors bupropion. } 1 \text { SR reported a } \\
\text { combined } 3+\text { week abstinence RR } \\
\text { of } 1.63 \text { ( } 95 \% \text { CI } 1.02 \text { to } 2.59) \text {. }\end{array}$ & Low & $\begin{array}{l}\text { Small body of evidence } \\
\text { Imprecise estimates }\end{array}$ \\
\hline Use & & $\begin{array}{l}\text { No difference. Use of cocaine, } \\
\text { combined SMD } 0.24(95 \% \\
\text { CI }-0.06 \text { to } 0.54) \text {. }\end{array}$ & Low & \\
\hline Retention & $1 \mathrm{SR}$ of $3 \mathrm{RCTs}^{17}(N=325)$ & $\begin{array}{l}\text { No difference. The SR's } \\
\text { combined RR for participants not } \\
\text { completing the trial was } 0.99 \\
(95 \% \text { CI } 0.79 \text { to } 1.25 \text { ). }\end{array}$ & Moderate & Inconsistent results \\
\hline Harms & $1 \mathrm{SR}$ of $1 \mathrm{RCT}^{7}$ & $\begin{array}{l}\text { No difference. Mean withdrawals } \\
\text { due to AEs RD } 0.00(95 \% \\
\text { CI }-0.05 \text { to. } 0.05) \\
\text { SAE: NA }\end{array}$ & $\begin{array}{l}\text { Insufficient } \\
\text { No evidence: } \\
\text { SAE }\end{array}$ & Small body of evidence \\
\hline \multicolumn{5}{|c|}{ Antipsychotics (all) } \\
\hline Abstinence & $1 \mathrm{SR}$ of $3 \mathrm{RCTs}^{8}(N=139)$ & $\begin{array}{l}\text { No difference. } 1 \text { SR reported a } \\
\text { combined } 3+\text { week abstinence RR } \\
\text { of } 1.30(95 \% \text { CI } 0.73 \text { to } 2.32) \text {. }\end{array}$ & Low & $\begin{array}{l}\text { Small body of evidence } \\
\text { Imprecise estimate }\end{array}$ \\
\hline Use & $\begin{array}{l}1 \mathrm{SR} \text { of } 2 \mathrm{RCTs}^{8}(N=150) \\
1 \text { high-ROB RCT }{ }^{22}(N=18 \text { opioid } \\
\text { randomized, } 41 \text { enrolled } \\
\text { opioid-dependent participants })\end{array}$ & No difference. & Low & $\begin{array}{l}\text { Small body of evidence } \\
\text { Methodological limitations of } \\
\text { studies. Indirectness of population. }\end{array}$ \\
\hline $\begin{array}{l}\text { Relapse } \\
\text { Lapse }\end{array}$ & $\begin{array}{l}1 \text { high-ROB } \mathrm{RCT}^{52}(N=18 \text { opioid } \\
\text { randomized, } 41 \text { enrolled } \\
\text { opioid-dependent participants })\end{array}$ & $\begin{array}{l}\text { No difference. } \\
\text { No difference. }\end{array}$ & $\begin{array}{l}\text { Insufficient } \\
\text { Insufficient }\end{array}$ & $\begin{array}{l}\text { Small, methodologically limited } \\
\text { single trial. Indirectness (of results } \\
\text { to general population-participants } \\
\text { had achieved abstinence prior to the } \\
\text { outpatient phase). Lapse is defined } \\
\text { as the first cocaine positive UDS, } \\
\text { relapse is } 2 \text { consecutive cocaine } \\
\text { positive UDS'. }\end{array}$ \\
\hline Retention & $\begin{array}{l}1 \mathrm{SR} \text { of } 8 \mathrm{RCTs}^{8}(N=397) \\
1 \text { high-ROB RCT }{ }^{22}(N=18 \\
\text { randomized, } 41 \text { enrolled } \\
\text { opioid-dependent participants })\end{array}$ & $\begin{array}{l}\text { Favors any antipsychotic. } 1 \text { SR } \\
\text { reported dropouts RR } 0.75 \text { (95\% } \\
\text { CI } 0.57 \text { to 0.97). } \\
1 \text { high-ROB RCT of comorbid } \\
\text { cocaine and opioid-dependent } \\
\text { methadone-maintained partici- } \\
\text { pants found no difference in } \\
\text { retention between groups. }\end{array}$ & Moderate & $\begin{array}{l}\text { Newer trial found no difference } \\
\text { (indirectness of population). }\end{array}$ \\
\hline
\end{tabular}


Table 2. (continued)

\begin{tabular}{|c|c|c|c|c|}
\hline Outcomes & $\begin{array}{l}N \text { studies per outcome; ROB } \\
(N=\text { combined participants })\end{array}$ & Summary of findings by outcome & $\begin{array}{l}\text { Strength of } \\
\text { evidence* }\end{array}$ & $\begin{array}{l}\text { Comments and rationale for } \\
\text { strength of evidence rating }\end{array}$ \\
\hline Harms & $\begin{array}{l}1 \text { high-ROB } \mathrm{RCT}^{22}(N=18 \\
\text { randomized, } 41 \text { enrolled } \\
\text { opioid-dependent participants })\end{array}$ & $\begin{array}{l}\text { Withdrawals: no difference. } \\
\text { SAE: NA }\end{array}$ & $\begin{array}{l}\text { Insufficient } \\
\text { No evidence: } \\
\text { SAE }\end{array}$ & $\begin{array}{l}\text { Small, methodologically limited } \\
\text { single trial. Indirectness (of } \\
\text { population) }\end{array}$ \\
\hline \multicolumn{5}{|c|}{ Antipsychotics (first generation): haloperidol } \\
\hline Abstinence & $\mathrm{NA}$ & NA & No evidence & NA \\
\hline Use & NA & NA & No evidence & NA \\
\hline Retention & $1 \mathrm{SR}$ of $1 \mathrm{RCT}^{8}(N=31)$ & $\begin{array}{l}\text { No difference. } 1 \text { SR reported a } \\
\text { RR for participants not } \\
\text { completing the trial of } 1.50 \text { ( } 95 \% \\
\text { CI } 0.63 \text { to } 3.57) \text {. } 1 \text { head to head } \\
\text { trial found no difference between } \\
\text { haloperidol and olanzapine }(N= \\
31 \text {; RR } 1.50,95 \% \text { CI } 0.63 \text { to } \\
3.57) \text {. }\end{array}$ & Insufficient & $\begin{array}{l}\text { Findings are from a single study in } \\
\text { a SR/meta-analysis of } 14 \text { RCTs. }\end{array}$ \\
\hline Harms & NA & NA & No evidence & NA \\
\hline \multicolumn{5}{|c|}{ Antipsychotics (second generation): aripiprazole, olanzapine, risperidone, quetiapine } \\
\hline Abstinence & $1 \mathrm{SR}$ of $3 \mathrm{RCTs}^{8}(\mathrm{~N}=139)$ & $\begin{array}{l}\text { No difference. Three studies in a } \\
\text { SR found no difference between } \\
\text { an atypical antipsychotic and } \\
\text { placebo on sustained abstinence. }\end{array}$ & Low & $\begin{array}{l}\text { Small body of evidence } \\
\text { Imprecise estimate }\end{array}$ \\
\hline Use & $\begin{array}{l}1 \mathrm{SR} \text { of } 1 \mathrm{RCT}^{8}(N=31) \\
1 \text { high-ROB RCT }{ }^{22}(N=18 \\
\text { randomized, } 41 \text { enrolled } \\
\text { opioid-dependent participants })\end{array}$ & $\begin{array}{l}\text { No difference. } 1 \text { RCT from } 1 \text { SR } \\
\text { and } 1 \text { high-ROB RCT of opioid- } \\
\text { dependent participants found no } \\
\text { difference between groups. }\end{array}$ & Insufficient & $\begin{array}{l}\text { Small body of evidence } \\
\text { Methodological limitations of } \\
\text { studies. Indirectness of population. }\end{array}$ \\
\hline Relapse & $\begin{array}{l}1 \text { high-ROB RCT }{ }^{22}(N=18 \\
\text { randomized, } 41 \text { enrolled }\end{array}$ & $\begin{array}{l}\text { No difference. } 1 \text { high-ROB found } \\
\text { no difference in relapse by group. }\end{array}$ & Insufficient & $\begin{array}{l}\text { Small, methodologically limited } \\
\text { single trial. Indirectness (of results }\end{array}$ \\
\hline Lapse & opioid-dependent participants) & $\begin{array}{l}\text { No difference. } 1 \text { high-ROB found } \\
\text { no difference in lapse by group. }\end{array}$ & Insufficient & $\begin{array}{l}\text { to general population-participants } \\
\text { had achieved abstinence prior to the } \\
\text { outpatient phase). Lapse is defined } \\
\text { as the first cocaine positive UDS, } \\
\text { relapse is } 2 \text { consecutive cocaine } \\
\text { positive UDS'. }\end{array}$ \\
\hline Retention & $\begin{array}{l}1 \text { SR of } 7 \mathrm{RCT}^{8}(N=365) \\
1 \text { high-ROB RCT }{ }^{22}(N=18 \\
\text { randomized, } 41 \text { enrolled }\end{array}$ & $\begin{array}{l}\text { No difference. Seven studies in } 1 \\
\text { SR and } 1 \text { high-ROB RCT of } \\
\text { comorbid cocaine and opioid- }\end{array}$ & Moderate & $\begin{array}{l}\text { Newer trial found no difference } \\
\text { (indirectness of population). }\end{array}$ \\
\hline
\end{tabular}

comorbid cocaine and opioiddependent methadone-maintained participants found no benefit of atypical antipsychotics on study retention

Harms 1 high-ROB RCT ${ }^{22}(N=18$ randomized, 41 enrolled opioid-dependent participants)

\begin{tabular}{|c|c|}
\hline \multicolumn{2}{|c|}{ Antipsychotics (other): reserpine } \\
\hline Abstinence & NA \\
\hline Use & $1 \mathrm{SR}$ of $1 \mathrm{RCT}^{8}(N=119)$ \\
\hline Retention & NA \\
\hline Harms & NA \\
\hline \multicolumn{2}{|c|}{ Anxiolytics: buspirone } \\
\hline Abstinence & 1 High-ROB RCT $^{26}(N=62)$ \\
\hline
\end{tabular}

Retention

Withdrawal

due to $\mathrm{AE}$

Severe AE

Cognitive enhancing drugs: memantine, atomoxetine Abstinence 1 low-ROB RCT ${ }^{24}(N=81)$
No difference. 1 high-ROB RCT of comorbid cocaine and opioiddependent methadone-maintained participants found no difference in withdrawals due to AEs by group. SAE: NA

NA

No difference. 1 study in the SR found a no difference in use between groups.

NA

NA

No difference. 1 RCT found no difference between groups in the mean number of days of (post-discharge) abstinence. NA

No difference. 1 RCT found no difference between groups in number of days to lapse.

No difference. 1 RCT reported high rates of retention $(94 \%$ buspirone vs $93 \%$ placebo), but no difference between groups. were no withdrawals due to AEs. Favors placebo. In 1 RCT there were 3 SAEs in participants receiving buspirone vs 0 receiving placebo. No difference. In 1 RCT there
Insufficient

No evidence:

SAE

No evidence

Insufficient

No evidence

No evidence

Insufficient

No evidence

Insufficient

Insufficient

Insufficient

Insufficient
Small, methodologically limited single trial. Indirectness (of results to general population - participants had achieved abstinence prior to the outpatient phase).

NA

Small body of evidence. Imprecise estimate.

NA

NA

Small, methodologically limited single trial. Indirectness (of results to general population - participants had achieved abstinence prior to the outpatient phase). Lapse is defined as the first cocaine positive UDS, relapse is 2 consecutive cocaine positive UDS'. 
Table 2. (continued)

\begin{tabular}{|c|c|c|c|c|}
\hline Outcomes & $\begin{array}{l}N \text { studies per outcome; ROB } \\
(N=\text { combined participants })\end{array}$ & Summary of findings by outcome & $\begin{array}{l}\text { Strength of } \\
\text { evidence* }\end{array}$ & $\begin{array}{l}\text { Comments and rationale for } \\
\text { strength of evidence rating }\end{array}$ \\
\hline & & $\begin{array}{l}\text { No difference. Participants who } \\
\text { did not achieve abstinence at } \\
\text { baseline }(N=45) \text {, there was no } \\
\text { difference between groups in the } \\
\text { achievement of sustained } \\
\text { abstinence ( } 3+\text { weeks). }\end{array}$ & & $\begin{array}{l}\text { Single small RCT with a } 2 \text {-week } \\
\text { placebo lead-in to encourage absti- } \\
\text { nence after randomization. }\end{array}$ \\
\hline Use & $\begin{array}{l}1 \text { low-ROB RCT }{ }^{24}(N=81) \\
1 \text { unclear-ROB } \operatorname{RCT}^{25}(N=50)\end{array}$ & $\begin{array}{l}\text { No difference. There was no } \\
\text { difference in cocaine negative } \\
\text { UDS' between groups. }\end{array}$ & Insufficient & \multirow{4}{*}{$\begin{array}{l}\text { Small body of evidence. } \\
\text { Methodological limitations of } \\
\text { studies. } \\
\text { Small body of evidence. } \\
\text { Indirectness (of results to general } \\
\text { population - participants had } \\
\text { achieved abstinence prior to the } \\
\text { outpatient phase). Relapse is de- } \\
\text { fined as } 2 \text { consecutive cocaine } \\
\text { positive UDS'. } \\
\text { Small body of evidence. } \\
\text { Methodological limitations of } \\
\text { studies. }\end{array}$} \\
\hline Relapse & 1 low-ROB RCT ${ }^{24}(N=81)$ & $\begin{array}{l}\text { No difference. Among } \\
\text { participants who achieved } \\
\text { abstinence at baseline }(N=36) \text {, } \\
\text { there was no difference between } \\
\text { groups in relapse or time to } \\
\text { relapse. }\end{array}$ & Insufficient & \\
\hline Retention & 1 low-ROB RCT ${ }^{24}(N=81)$ & \multirow{2}{*}{$\begin{array}{l}\text { No difference. There was no } \\
\text { difference in retention by group. } \\
\text { No difference. There was no } \\
\text { difference in retention by group. } \\
\text { No difference. } 0 \text { participants } \\
\text { receiving memantine experienced } \\
\text { a SAE compared with } 2 \text { who } \\
\text { received placebo. }\end{array}$} & Insufficient & \\
\hline Harms & 1 unclear-ROB $\mathrm{RCT}^{25}(N=50)$ & & Insufficient & \\
\hline \multicolumn{5}{|c|}{$\begin{array}{l}\text { Psychostimulants: dexamphetamine, mazindol, methamphetamine, methylphenidate, mixed amphetamine salts, modafinil, lisdexamphetamine, } \\
\text { selegiline }\end{array}$} \\
\hline Abstinence & $1 \mathrm{SR}$ of 14 studies $^{7}(N=1549)$ & $\begin{array}{l}\text { Favors psychostimulants. } 1 \mathrm{SR} \\
\text { reported a combined } 3+\text { week } \\
\text { abstinence RR of } 1.36 \text { ( } 95 \% \text { CI } \\
1.05 \text { to } 1.77) \text {. }\end{array}$ & Low & $\begin{array}{l}\text { Large body of evidence and } \\
\text { consistent results even after } \\
\text { removing bupropion studies, but } \\
\text { many trials were methodologically } \\
\text { flawed. Findings from individual } \\
\text { drugs favor dexamphetamine (small } \\
\text { body of evidence) and mixed } \\
\text { amphetamine salts (single study). }\end{array}$ \\
\hline Use & $1 \mathrm{SR}$ of $8 \mathrm{RCTs}^{7}(N=526)$ & $\begin{array}{l}\text { No difference. Use of cocaine, } \\
\text { combined SMD } 0.16 \text { ( } 95 \% \text { CI - } \\
0.02 \text { to } 0.33) \text {. }\end{array}$ & Low & $\begin{array}{l}\text { Trend toward small benefit, } \\
\text { inconsistent results }\end{array}$ \\
\hline Retention & $1 \mathrm{SR}$ of 24 studies $^{7}(N=2205)$ & $\begin{array}{l}\text { No difference. Number of } \\
\text { participants who did not complete } \\
\text { the trial, combined RR } 1.00(95 \% \\
\text { CI } 0.93 \text { to } 1.06)\end{array}$ & Moderate & $\begin{array}{l}\text { Methodological limitations of many } \\
\text { included studies. Heterogeneous } \\
\text { population. } \\
\text { No bupropion studies are included }\end{array}$ \\
\hline Harms & $\begin{array}{l}\text { Withdrawal: } 1 \text { SR of } 19 \mathrm{RCTs}^{7} \\
(N=1601) \\
\text { Serious AEs: } 1 \mathrm{SR} \text { of } 6 \mathrm{RCTs}^{7} \\
(N=444)\end{array}$ & $\begin{array}{l}\text { No difference. Number of } \\
\text { participants who withdrew due to } \\
\text { AEs, combined mean RD } 0.00 \\
\text { (95\% CI }-0.01 \text { to } 0.01) \text {. } \\
\text { No difference. Number of } \\
\text { participants who reported severe } \\
\text { AEs, combined mean RD }-0.02 \\
(95 \% \text { CI }-0.06 \text { to } 0.01) \text {. }\end{array}$ & Moderate & in findings of SAEs. \\
\hline \multicolumn{5}{|c|}{ Anticonvulsants and muscle relaxants } \\
\hline Abstinence & 2 unclear-ROB RCTs ${ }^{27,28}(N=230)$ & No difference. & Low & \\
\hline Use & 2 unclear-ROB RCTs $27,28(N=230)$ & No difference. & Low & \\
\hline Retention & 2 unclear-ROB RCTs $27,28(N=230)$ & No difference. & Low & \\
\hline $\begin{array}{l}\text { Withdrawal } \\
\text { due to } \mathrm{AE}\end{array}$ & 1 unclear-ROB $\operatorname{RCT}^{27}(N=70)$ & No difference. & Insufficient & \\
\hline Severe AE & $\begin{array}{l}2 \text { unclear-ROB RCTs }{ }^{27,}, 28 \\
(\mathrm{~N}=230)\end{array}$ & No difference. & Low & \\
\hline \multicolumn{5}{|c|}{ Carbamazepine, gabapentin, lamotrigine, phenytoin, tiagabine, topiramate, and vigabatrin (drugs combined in analysis) } \\
\hline Abstinence & $1 \mathrm{SR}^{5}$ & & No evidence & These represent the combined \\
\hline Use & $1 \mathrm{SR}$ of $9 \mathrm{RCTs}^{5}(N=867)$ & $\begin{array}{l}\text { No difference. Use of cocaine } \\
\text { (self-reported or objective), } \\
\text { combined RR } 0.92(95 \% \\
\text { CI } 0.84 \text { to } 1.02)^{5}\end{array}$ & Moderate ${ }^{5}$ & $\begin{array}{l}\text { results for all drug classes included } \\
\text { in the SR. } \\
\text { SOE was determined by the SR } \\
\text { authors }\end{array}$ \\
\hline Retention & $\begin{array}{l}\text { 1 SR that included } 17 \mathrm{RCTs}^{5} \\
(N=1695)\end{array}$ & $\begin{array}{l}\text { No difference. RR } 0.95(95 \% \text { CI } \\
0.86 \text { to } 1.05)^{5}\end{array}$ & Moderate $e^{5}$ & \\
\hline $\begin{array}{l}\text { Topiramate } \\
\text { Abstinence }\end{array}$ & $\begin{array}{l}1 \text { low-ROB RCT }{ }^{29}(N=60) \\
2 \text { unclear-ROB RCTs }\end{array}$ & $\begin{array}{l}\text { Favors topiramate ( } 3 \text { RCTs). } \\
\text { Relapse prevention RCTs: } \\
\text { combined findings from } 2 \\
\text { unclear-ROB RCTs }{ }^{31,32} \text { (RR } \\
2.56 \text { [95\% CI } 1.39 \text { to } 4.73] \text { ) for } 3 \\
\text { or more weeks of continuous } \\
\text { abstinence }\end{array}$ & Low & \\
\hline
\end{tabular}


Table 2. (continued)

\begin{tabular}{|c|c|c|c|c|}
\hline Outcomes & $\begin{array}{l}N \text { studies per outcome; ROB } \\
\text { ( } N=\text { combined participants) }\end{array}$ & Summary of findings by outcome & $\begin{array}{l}\text { Strength of } \\
\text { evidence* }\end{array}$ & $\begin{array}{l}\text { Comments and rationale for } \\
\text { strength of evidence rating }\end{array}$ \\
\hline Use & 1 low-ROB $\mathrm{RCT}^{29}(N=60)$ & Favors topiramate. & Insufficient & Only 1 small trial \\
\hline Retention & $\begin{array}{l}5 \text { RCTs: } 1 \text { high-ROB } \\
2 \text { unclear-ROB }{ }^{30,32} ; \\
2 \text { low-ROB }{ }^{29,}, 34(N=617)\end{array}$ & $\begin{array}{l}\text { No difference. Combined RR } \\
1.01 \text { (95\% CI: } 0.93 \text { to } 1.10 \text { ). }\end{array}$ & Moderate & $\begin{array}{l}\text { Methodological limitations of } \\
\text { several trials. }\end{array}$ \\
\hline Harms & 1 low-ROB $\operatorname{RCT}^{29}(N=60)$ & $\begin{array}{l}\text { No withdrawals occurred due to } \\
\text { AE. No severe AEs occurred. }\end{array}$ & Insufficient & Only 1 small RCT \\
\hline \multicolumn{5}{|c|}{ - } \\
\hline Abstinence & 1 high-ROB $\mathrm{RCT}^{83}(N=103)$ & $\begin{array}{l}\text { Favors vigabatrin. Full 3-week } \\
\text { end-of-trial abstinence } 28 \% \text { vs } \\
7.5 \% P \leq 0.01\end{array}$ & Insufficient & $\begin{array}{l}\text { Incomplete data was reported for } \\
\text { the full trial period. }\end{array}$ \\
\hline Use & $\begin{array}{l}1 \text { unclear-ROB RCT }{ }^{84}(N=186) \\
1 \text { high-ROB RCT }{ }^{83}(N=103)\end{array}$ & $\begin{array}{l}\text { No difference. Total events: } 76 \\
\text { (treatment), } 86 \text { (placebo). RR } \\
0.88 ; 95 \% \text { CI } 0.69 \text { to } 1.13\end{array}$ & Low & Analysis from $\mathrm{SR}^{5}$ \\
\hline Retention & $\begin{array}{l}1 \text { unclear-ROB } \operatorname{RCT}^{84}(N=186) \\
1 \text { high-ROB } \operatorname{RCT}^{83}(N=103)\end{array}$ & $\begin{array}{l}\text { No difference. Total events: } 98 \\
\text { (treatment), } 108 \text { (placebo). RR }\end{array}$ & Low & \\
\hline Harms & 1 unclear-ROB $\operatorname{RCT}^{84}(N=186)$ & $\begin{array}{l}\text { No difference. RR } 0.97 ; 95 \% \text { CI } \\
0.88 \text { to } 1.08\end{array}$ & Insufficient & \\
\hline \multicolumn{5}{|c|}{ Medications FDA-approved for other substance use disorders } \\
\hline \multicolumn{5}{|c|}{ Acamprosate } \\
\hline Abstinence & - & No evidence & - & \\
\hline Use & 1 low-ROB $\mathrm{RCT}^{50}(N=60)$ & $\begin{array}{l}\text { No difference. } \% \text { UDS(-): } 22 \% \\
\text { vs } 23 \%, P=0.44\end{array}$ & Insufficient & Only 1 small RCT \\
\hline Retention & 1 low-ROB $\operatorname{RCT}^{50}(N=60)$ & $\begin{array}{l}\text { No difference. } 18 / 34(53 \%) \text { vs } 18 / \\
26(69 \%), P=N S\end{array}$ & Insufficient & Only 1 small RCT \\
\hline Harms & - & No evidence & - & \\
\hline \multicolumn{5}{|c|}{ Buprenorphine plus naloxone, 2 doses } \\
\hline Abstinence & 1 low-ROB RCT ${ }^{49}(N=302)$ & $\begin{array}{l}\text { No difference. Rates of abstinence } \\
\text { during weeks } 5-8 \text { similar between } \\
\text { placebo group }(16 \%) \text { and Bup } \\
4 \mathrm{mg} 17.9 \%,(P=0.36) \text { and Bup } \\
16 \mathrm{mg} 18.6 \%,(P=0.32)\end{array}$ & Insufficient & Only 1 trial \\
\hline Use & 1 low-ROB RCT ${ }^{49}(N=302)$ & $\begin{array}{l}\text { Mixed findings. Significantly less } \\
\text { use with Bup } 16 \mathrm{mg}+\text { naloxone } \\
4 \mathrm{mg} \text { vs placebo. No difference } \\
\text { with lower dose }\end{array}$ & Insufficient & \\
\hline Retention & 1 low-ROB RCT ${ }^{49}(N=302)$ & $\begin{array}{l}\text { No difference. Rates of retention } \\
\text { similar between placebo }(87.3 \%) \\
\text { vs Bup } 4 \mathrm{mg}(86.0 \%) \text { vs Bup } \\
16 \mathrm{mg}(88.0 \%)\end{array}$ & Insufficient & \\
\hline Harms & - & No evidence & - & \\
\hline \multicolumn{5}{|c|}{ Buprenorphine vs methadone } \\
\hline Abstinence & 2 low-ROB RCTs ${ }^{35,36}(N=278)$ & $\begin{array}{l}\text { Mixed findings. Longer } \\
\text { abstinence with methadone in } 1 \\
\text { RCT; no difference in } 1 \text { RCT }\end{array}$ & Insufficient & Mixed findings \\
\hline Use & 1 low-ROB RCT ${ }^{35}(N=116)$ & $\begin{array}{l}\text { Favors Methadone. Lower use } \\
\text { with methadone vs buprenorphine } \\
(P<0.05)\end{array}$ & Insufficient & \\
\hline Retention & 2 low-ROB RCTs ${ }^{35,36}(N=278)$ & $\begin{array}{l}\text { Mixed findings. Better retention } \\
\text { with methadone in } 1 \text { RCT; no } \\
\text { difference in } 1 \text { RCT }\end{array}$ & Insufficient & Mixed findings \\
\hline $\begin{array}{l}\text { Harms } \\
\text { Disulfiram }\end{array}$ & 1 low-ROB RCT ${ }^{36}(N=162)$ & Elevated LFT in 1 subject & Insufficient & \\
\hline $\begin{array}{l}\text { Disulfiram } \\
\text { Abstinence }\end{array}$ & $3 \mathrm{RCTs}^{37,41,85}(N=296)$ & $\begin{array}{l}\text { No difference. Continuous } \\
\text { abstinence disulfiram vs placebo, } \\
\text { combined RR from } 3 \text { RCTs } N= \\
\text { 296, RR } 0.96 \text { ( } 95 \% \text { CI } 0.63 \text { to } \\
1.45 \text { ) }\end{array}$ & Low & ROB unclear overall \\
\hline Use & $\begin{array}{l}4 \mathrm{RCTs}^{37-40} \\
(N=440)\end{array}$ & $\begin{array}{l}\text { No difference. Combined RR } \\
\text { from } 4 \text { RCTs: } 0.95 \text { ( } 95 \% \text { CI } 0.64 \\
\text { to } 1.39 \text { ). The effect varied among } \\
\text { studies, and statistical } \\
\text { heterogeneity was highly } \\
\text { significant }(P<0.001) \text {. }\end{array}$ & Low & $\begin{array}{l}\text { Heterogeneous findings among } \\
\text { studies }\end{array}$ \\
\hline Retention & $\begin{array}{l}1 \mathrm{SR}^{4} \text { that included } 2 \mathrm{RCTs} \\
(N=87): 1 \text { unclear-ROB }(N=20),{ }^{85} \\
1 \text { high-ROB }{ }^{86}(N=67) \\
5 \text { low-ROB RCTs }{ }^{37-41}(N=617)\end{array}$ & $\begin{array}{l}\text { Favors placebo. Treatment } \\
\text { retention was lower with } \\
\text { disulfiram: Meta-analysis of } 7 \\
\text { RCTs, } N=704 \text {, RR } 0.90 \text { (95\% CI } \\
0.83 \text { to } 0.99 \text { ). }\end{array}$ & Moderate & $\begin{array}{l}\text { The combination of findings from } \\
\text { all } 7 \text { studies }(N=704) \text { was } \\
\text { statistically homogeneous }(P=0.90)\end{array}$ \\
\hline Harms & $4 \mathrm{RCTs}^{38-41}(N=548)$ & $\begin{array}{l}\text { Withdrawals due to AE ranged } \\
\text { from } 0 \text { to } 5.9 \% \text {, and included } \\
\text { elevated liver enzymes and rash. } \\
\text { Severe AEs not otherwise reported. }\end{array}$ & Low & - \\
\hline
\end{tabular}


Table 2. (continued)

\begin{tabular}{|c|c|c|c|c|}
\hline Outcomes & $\begin{array}{l}N \text { studies per outcome; ROB } \\
(N=\text { combined participants) }\end{array}$ & Summary of findings by outcome & $\begin{array}{l}\text { Strength of } \\
\text { evidence** }\end{array}$ & $\begin{array}{l}\text { Comments and rationale for } \\
\text { strength of evidence rating }\end{array}$ \\
\hline \multicolumn{5}{|l|}{ Naltrexone } \\
\hline Abstinence & $\begin{array}{l}2 \text { low-ROB RCT }{ }^{43,} 47 \\
1 \text { unclear-ROB RCT }{ }^{46} \\
(N=416)\end{array}$ & $\begin{array}{l}\text { No difference. } 2 \text { studies found no } \\
\text { differences in } N \text { weeks to relapse. } \\
1 \text { study found no differences in } \\
\text { abstinence ( } 17.9 \% \text { vs } 17.1 \%, P= \\
0.918 \text { ) }\end{array}$ & Low & $\begin{array}{l}\text { Imprecision due to small number of } \\
\text { studies; } 1 \text { study rated unclear ROB }\end{array}$ \\
\hline Use & 1 low-ROB $\mathrm{RCT}^{45}(N=80)$ & $\begin{array}{l}\text { No difference. } 1 \text { study compared } \\
\%(+) \text { UDS at weeks } 1-4 ; 5-8 \text {; } \\
\text { and } 9-12 \text { and found no differ- } \\
\text { ences between T vs C. }\end{array}$ & Insufficient & Only 1 small RCT \\
\hline Retention & $\begin{array}{l}3 \text { low RCTs }{ }^{44,45,48} \\
1 \text { unclear-ROB RCT }{ }^{46}(N=416)\end{array}$ & $\begin{array}{l}\text { No difference. All } 4 \text { studies } \\
\text { reported no differences in } \\
\text { treatment retention }\end{array}$ & Low & $\begin{array}{l}\text { Imprecision due small number of } \\
\text { trials; indirectness due to behavioral } \\
\text { co-interventions }\end{array}$ \\
\hline Harms & 1 low $\operatorname{RCT}^{47}(N=64)$ & $\begin{array}{l}\text { No difference. In } 1 \text { trial of } 64 \text { pts., } \\
2 \text { in treatment arm and } 11 \text { in } \\
\text { placebo arm experienced AE, } \\
\text { non-significant. }\end{array}$ & Insufficient & Only 1 small RCT \\
\hline \multicolumn{5}{|c|}{ 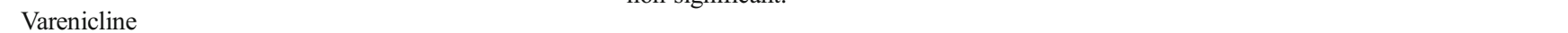 } \\
\hline Abstinence & - & No evidence & - & \\
\hline Use & 2 unclear-ROB RCTs ${ }^{51,52}(N=68)$ & $\begin{array}{l}\text { No evidence. } 1 \text { study found trend } \\
\text { toward lower use with varenicline } \\
(\mathrm{OR}=0.49, P=0.08) ; 1 \text { study } \\
\text { found no difference }(P=0.84)\end{array}$ & Insufficient & $\begin{array}{l}\text { Few trials included; inconsistency } \\
\text { of findings }\end{array}$ \\
\hline Retention & 2 unclear-ROB RCTs ${ }^{51,52}(N=68)$ & $\begin{array}{l}\text { No difference. } 1 \text { study reported } \\
77 \% \text { total retention with no } \\
\text { "significant difference in time to } \\
\text { last visit" }(P=0.1) ; 1 \text { study } \\
\text { reported } 5 \text { drop out and no } \\
\text { differences between groups ( } P= \\
0.26 \text { ) }\end{array}$ & Insufficient & $\begin{array}{l}\text { Unclear risk of bias, small number } \\
\text { of studies. }\end{array}$ \\
\hline Harms & 1 unclear-ROB RCT ${ }^{51}(N=31)$ & $\begin{array}{l}\text { No difference. } 1 \text { trial reported no } \\
\text { withdrawals due to AEs. }\end{array}$ & Insufficient & $\begin{array}{l}\text { Unclear risk of bias, only } 1 \text { trial, } \\
\text { few evens }\end{array}$ \\
\hline \multicolumn{5}{|c|}{ Dopamine agonists } \\
\hline Abstinence & $1 \mathrm{SR}$ of $11 \operatorname{RCTs}^{6}(N=731)$ & $\begin{array}{l}\text { No difference. At } 6 \text { weeks: RR } \\
1.12(95 \% \text { CI } 0.85 \text { to } 1.47) \text {; at } \\
4 \text { months: RR } 1.1 \text { ( } 95 \% \text { CI } 0.61 \\
\text { to } 1.98)\end{array}$ & Low 6 & $\begin{array}{l}\text { Strength of evidence was } \\
\text { determined by the SR authors }\end{array}$ \\
\hline Use & NR & NR & - $\quad$ & \\
\hline Retention & 1 SR of 20 studies $^{6}(N=1656)$ & $\begin{array}{l}\text { No difference. RR } 1.04 \text { (95\% CI } \\
0.94 \text { to } 1.14 \text { ) }\end{array}$ & Moderate $^{6}$ & \\
\hline Harms & 1 SR of 7 studies $^{6}(N=252)$ & $\begin{array}{l}\text { SAEs and withdrawals due to AE } \\
\text { NR. }\end{array}$ & No evidence ${ }^{6}$ & \\
\hline
\end{tabular}

AE, adverse event; CI, confidence interval; DSM, Diagnostic and Statistical Manual of Mental Disorders; MD, mean difference; NR, not reported; $P$, $p$ value; $R C T$, randomized control trial; $R D$, risk difference; $R R$, relative risk; ROB, risk of bias; SAE, severe adverse event; SMD, standard mean difference; SSRI, selective serotonin reuptake inhibitor; SNRI, serotonin and norepinephrine reuptake inhibitor; SR, systematic review; UDS, urine drug screens

*The overall quality of evidence for each outcome is based on the consistency, coherence, and applicability of the body of evidence, as well as the internal validity of individual studies. The strength of evidence is classified as follows ${ }^{16}$ : high, further research is very unlikely to change our confidence on the estimate of effect; moderate, further research is likely to have an important impact on our confidence in the estimate of effect and may change the estimate; low, further research is very likely to have an important impact on our confidence in the estimate of effect and is likely to change the estimate; insufficient, any estimate of effect is very uncertain

moderate SOE; Fig. 2). We found insufficient evidence related to harms.

Antipsychotics: Aripiprazole, Haloperidol, Lamotrigine, Olanzapine, Quetiapine, Risperidone, Reserpine. Fourteen RCTs $(N=719)$ in an existing $\mathrm{SR}^{8}$ and one additional $\mathrm{RCT}^{22}$ examined antipsychotics as a class for the treatment of cocaine use disorder. The additional $\mathrm{RCT}^{22}$ of recently abstinent subjects found no difference between $15 \mathrm{mg}$ of aripiprazole and placebo for any outcome of interest. Overall, we found low SOE that antipsychotics did not improve abstinence ${ }^{8}$ or reduce cocaine use, ${ }^{8,22}$ and insufficient evidence for lapse and relapse in participants abstinent at baseline. ${ }^{22}$ We found moderate SOE that antipsychotics improve study retention compared with placebo based on findings from eight RCTs in the SR (RR $0.75,95 \%$ CI 0.57 to 0.97$),{ }^{8}$ and the additional RCT. ${ }^{22}$ We found insufficient evidence to form conclusions on harms.

Psychostimulants: Dexamphetamine, Lisdexamfetamine, Mazindol, Methamphetamine, Methylphenidate, Mixed Amphetamine Salts, Modafinil, Selegiline. A SR of 14 RCTs examined psychostimulants for treatment of cocaine use disorder. These trials reported low-strength evidence that 


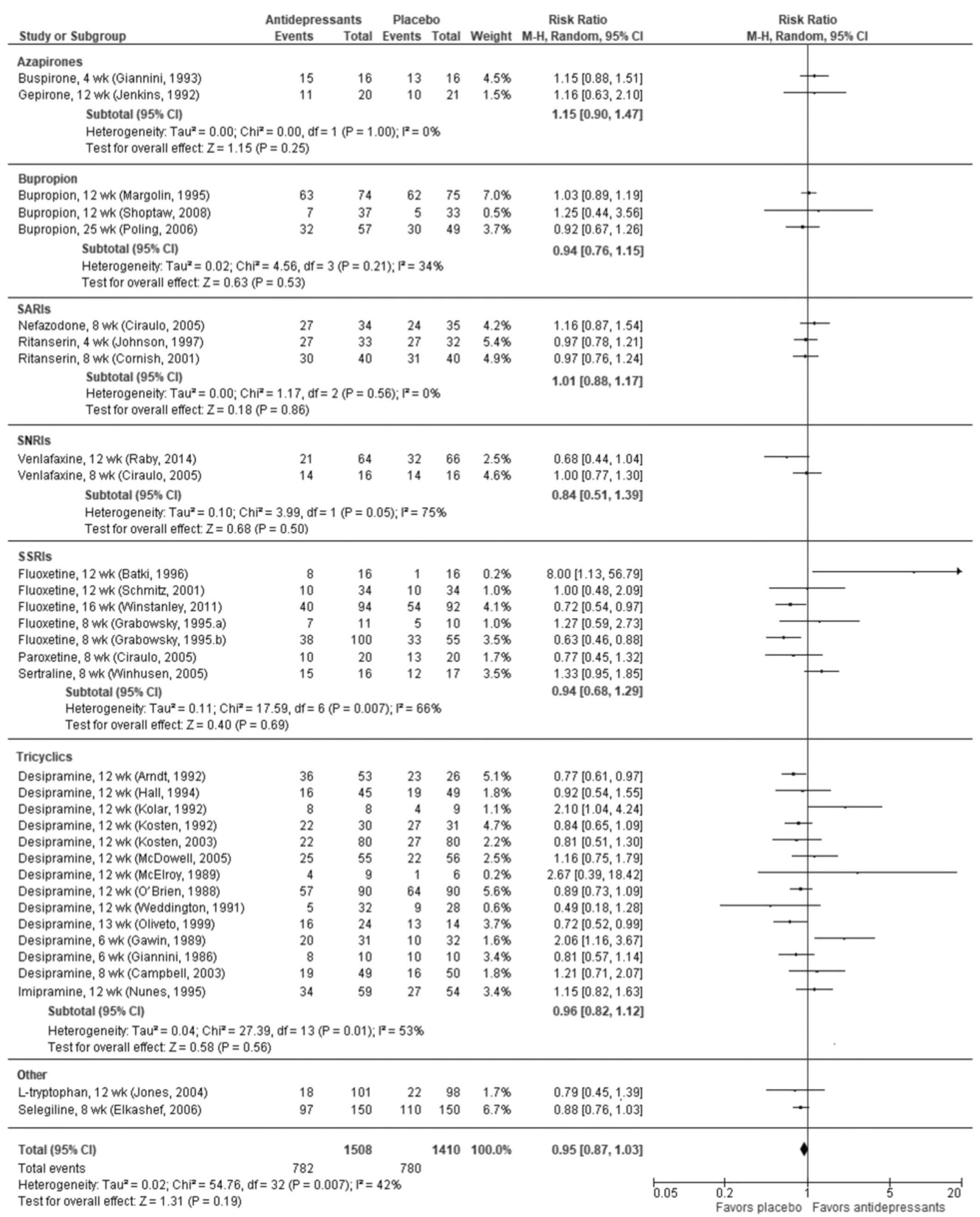

Figure 2 Treatment retention in RCTs of antidepressants vs placebo for cocaine use disorder.

psychostimulants improved abstinence versus placebo (RR $1.36,95 \%$ CI 1.05 to 1.77$))^{7}$ Although one study of bupropion (which we classified as an antidepressant) was included in the combined estimate, its removal does not change the 
conclusion. ${ }^{23}$ There were no significant differences between groups for cocaine use during the trial period (SOE low), study retention (moderate $\mathrm{SOE}$ ), or harms (moderate $\mathrm{SOE}$ ).

Cognitive Enhancing Drugs: Atomoxetine, Memantine. We found two small RCTs, including one examining memantine (low ROB) and the other examining atomoxetine (unclear ROB), that provide insufficient evidence to draw conclusions about the effects of cognitive enhancing drugs on any outcome of interest. ${ }^{24,25}$

Anxiolytics: Buspirone. We identified only one small $(N=$ 62), multi-site, high-ROB RCT that compared $60 \mathrm{mg}$ of buspirone to placebo, along with $\mathrm{CM}$ and once weekly optional individual or group psychosocial treatment and relapse prevention. ${ }^{26}$ This provides insufficient evidence for the use of buspirone for cocaine use disorder.

\section{Other Pharmacotherapies}

Anticonvulsants and Muscle Relaxants: Baclofen, Carbamazepine, Gabapentin, Lamotrigine, Phenytoin, Tiagabine, Topiramate, Vigabatrin. We identified 20 RCTs in a prior $\mathrm{SR},{ }^{5}$ and three additional $\mathrm{RCTs}^{27-29}$ examining the effectiveness of anticonvulsants and muscle relaxants. The SR examined anticonvulsants and found moderate SOE that anticonvulsants as a class are no different than placebo for retention (17 RCTs, combined RR $0.95,95 \%$ CI 0.86 to 1.05 ) or cocaine use ( 9 RCTs, RR $0.92,95 \%$ CI 0.84 to 1.02 ). We identified one additional RCT $(N=60)$ that found improved abstinence and a reduction in cocaine use associated with topiramate, and no difference in retention. ${ }^{29}$ Two additional $\mathrm{RCTs}^{27,28}$ compared baclofen (60 mg and $20 \mathrm{mg}$ ) to placebo. Neither study reported differences between groups on any of the outcomes of interest. Across all anticonvulsants and muscle relaxants as a class, there is insufficient evidence to form conclusions about the effects on abstinence, moderate SOE of no difference from placebo on cocaine use and study retention, and insufficient evidence on harms.

Topiramate. Five RCTs - four ${ }^{30-34}$ from an existing $\mathrm{SR}^{5}$ and one additional $\mathrm{RCT}^{29}$ - examined topiramate for cocaine use disorder. We found low SOE favoring topiramate over placebo for abstinence (2 RCTs, combined RR $2.56,95 \%$ CI, 1.39 to 4.73; Fig. 3) and moderate SOE that topiramate was no different from placebo for study retention (5 RCTs, combined RR $1.01,95 \%$ CI 0.93 to 1.10 ; Fig. 3). There was insufficient evidence to form conclusions on AEs.

Medications FDA-Approved for Other Substance Use Disorders: Acamprosate, Buprenorphine, Buprenorphine and Naloxone, Disulfiram, Methadone, Naltrexone, Varenicline. One $\mathrm{SR}^{4}$ and $18 \mathrm{RCTs}^{35-52}$ examined FDA-approved pharmacotherapies for other substance use disorders. We found low SOE from six trials ${ }^{43-48}$ that naltrexone was no different than placebo for abstinence or retention. There was insufficient evidence on use reduction and AEs. For studies of acamprosate, ${ }^{50}$ varenicline, ${ }^{51,52}$ buprenorphine plus naloxone, ${ }^{49}$ and methadone compared directly with buprenorphine, ${ }^{35,36}$ there was insufficient evidence to form conclusions on the outcomes of interest.

Disulfiram. Disulfiram for the treatment of cocaine use disorder was examined in a previous $\mathrm{SR}^{4}$ of seven RCTs and in five more recently published RCTs. ${ }^{37-41}$ There was low SOE that disulfiram does not increase abstinence (3 RCTs, combined RR $0.96,95 \%$ CI 0.63 to 1.45 ; Figure 7 ) or increase harms versus placebo. We found moderate SOE that disulfiram worsened rates of retention versus placebo (7 RCTs, combined RR 0.90, 95\% CI 0.83 to 0.99; Online Appendix Figure 5). The effects of disulfiram on cocaine use were significantly heterogeneous $\left(I^{2}=97 \%, P<0.00001\right)$ in a meta-analysis of four RCTs (Online Appendix Figure 5), and the evidence was therefore insufficient for drawing conclusions.

Dopamine Agonists: Amantadine, Bromocriptine, Cabergoline Hydergine, L-Dopa/Carbidopa, Pergolide, Pramipexole. A 2015 SR of 24 trials found no difference between dopamine agonists and placebo on retention (moderate SOE), abstinence (low SOE), and a lack of evidence on AEs. ${ }^{6}$ We identified no additional trials of examining dopamine agonists for cocaine use disorder.

Other Pharmacotherapies. Nineteen trials ${ }^{53-71}$ examined the effects of other drugs or drug combinations for cocaine use disorder (Table 2). Although there is insufficient evidence to form conclusions due to limited power, positive findings on abstinence and use reduction were reported in studies of doxazosin, ${ }^{59}$ ondansetron, ${ }^{66}$ propranolol,${ }^{70}$ and topiramate combined with mixed amphetamine salts. ${ }^{64}$ There were no positive or negative findings on the outcomes of interest for any of the other drugs or drug combinations.

Pharmacotherapies for Comorbid Opioid Use Disorder. Data from $6 \mathrm{SRs}^{4,5,7,8,17,72}$ and 14 additional RCTs ${ }^{22,35-41,49,51,}$ $54,61,62,69$ contribute to the evidence on pharmacotherapy for the treatment of cocaine use disorder in adults with comorbid opioid use disorder. Table 5 summarizes the findings of pharmacotherapies studied in patients with comorbid opioid use disorder, and additional details are provided in an online data supplement (Online Appendix Table 7).

We found low SOE that antidepressants are more effective than placebo for cocaine abstinence, ${ }^{7,} 17,72$ and that psychostimulants are more effective than placebo for reducing cocaine use in patients with comorbid opioid use disorder. ${ }^{8,22}$ However, we also found moderate SOE that both retention and dropouts due to AEs were higher in subjects receiving antidepressants versus placebo, and moderate $\mathrm{SOE}$ of poorer retention associated with disulfiram. There was no difference between placebo and antipsychotics or psychostimulants on 


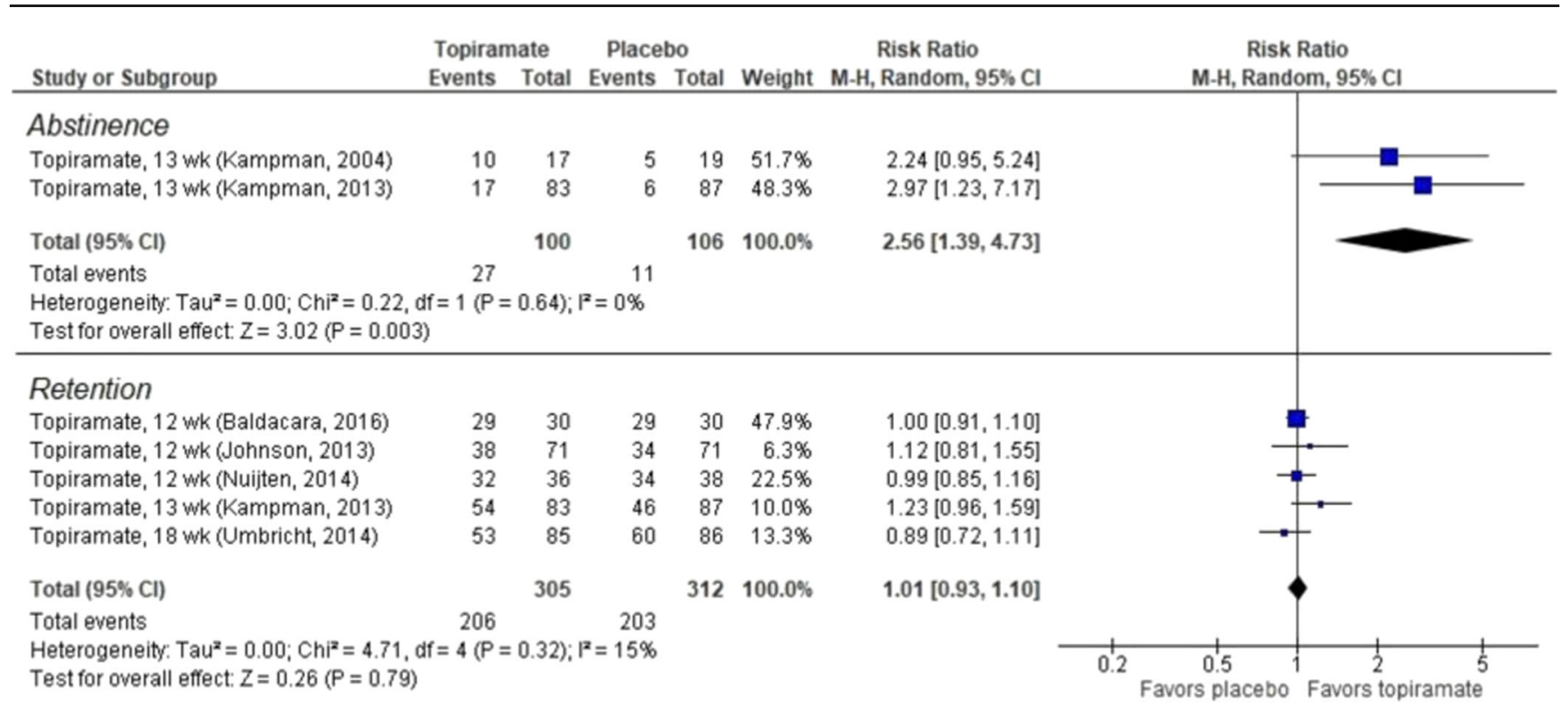

Figure 3 Abstinence and retention in RCTs of topiramate vs placebo for cocaine use disorder.

retention (low SOE). All other medication/outcomes were insufficient to form conclusions.

\section{DISCUSSION}

In this review, we identified seven SRs and 48 RCTs examining a variety of pharmacotherapies for cocaine use disorder. We found no strong evidence that any drug class was effective in increasing abstinence, reducing use, or improving retention rates for cocaine use disorder. However, we found low SOE that bupropion, psychostimulants, and topiramate may improve abstinence, and low SOE that sertraline may reduce relapse rates in abstinent patients. There was moderate SOE that antipsychotics may improve treatment retention. We also found moderate SOE that disulfiram may actually worsen treatment retention, and low SOE that SSRIs were associated with higher dropouts due to AEs (Table 4).

To our knowledge, this is the first report to summarize multiple classes of medications used in treatment of cocaine use disorder, which continues to be a global public health problem with increasing morbidity and mortality. ${ }^{73}$ One motivation for this review was to find potentially promising treatments and targets for future research for a devastating condition that has been historically difficult to treat with pharmacotherapy. Indeed, we did identify several promising treatments that may be good areas in which to prioritize future research (Table 3). Post hoc analyses in RCTs of bupropion suggest that it may be effective for patients with comorbid depression and in conjunction with $\mathrm{CM}$. We also found that psychostimulants - which serve as a form of agonist replacement therapy - may improve abstinence outcomes. Finally, we found that topiramate, thought to work via GABAergic pathways to regulate dopamine release, was potentially effective for abstinence and warrants continued exploration. ${ }^{74}$
Our review complements and extends the findings of prior SRs by examining and summarizing data across all drug classes. We defined abstinence as 2 or more weeks of negative UDS - which meant excluding studies using other measures of abstinence. We summarized retention as an outcome, recognizing that improving retention in treatment increases the chances for successful recovery of stimulant use disorders; therefore, we did not consider study retention in our SOE assessment. We were limited in our ability to compare and meta-analyze results across studies because many studies did not report these data, or used different measures, and future research should look to standardize outcome reporting such as 3 or more-week abstinence to compare efficacy across trials and drug classes. It is also possible that the lack of significant findings was due to insufficient power to detect differences.

A lack of engagement in treatment on the part of some study participants who are actively using stimulants may affect the efficacy of pharmacotherapies; retention rates varied widely across studies (24-97\%), and overall low rates of retention may have affected the assessment of treatment effectiveness in the majority of studies (attrition was greater than $20 \%$ in more than a third of the trials reporting retention rates). Unfortunately, pharmacotherapy alone (aside from antipsychotics) does not appear to be effective in improving treatment retention rates. Two areas of promise that are notable include those in which patients have already demonstrated engagement in treatment, or may have another rationale for ongoing engagement, as is the case for some patients with comorbid opiate use disorder. Indeed, we found low SOE that antidepressants and psychostimulants improved cocaine use outcomes in patients with comorbid opioid use disorder (Table 5). Given that the prevalence of cocaine use among heroin users is between 30 and $80 \%,{ }^{75}$ and concurrent opioid use increases risk of death due to cocaine, ${ }^{76}$ further investigation on treatments for comorbid opioid use disorder is warranted. 
Furthermore, more trials of medications that integrate evidence-based psychosocial and behavioral interventions are necessary to move the field forward. Given the largely disappointing pharmacotherapy results, these interventions (e.g., CM, CBT), alone or in combination, continue to be mainstays of treatment and management of stimulant use disorders. $^{77,78}$ A systematic review by Minozzi et al. found that any psychosocial treatment likely reduces dropout rates and may increase the period of abstinence (most of the studies reviewed included $\mathrm{CM}$ in addition to treatment as usual). ${ }^{78}$ The combination of pharmacotherapy with CM is an important area for future research as we do not know how medication may enhance the effectiveness of these interventions. ${ }^{79,} 80$ When we compared studies with a CM co-intervention to those without, we found that pharmacotherapeutic effects were similar in both. ${ }^{81}$

Our SR has several limitations. Our scope was broad, and we relied on existing SRs when available. We sought to minimize the disadvantages of using existing SRs by only including those that met key quality criteria; conducting updated searches to identify more recent trials; and combining data in meta-analysis from trials in previous SRs with newer trials from our search. Our definition of abstinence (3 or more weeks) served as a proxy for sustained abstinence, and the effects of treatment on long-term abstinence cannot be directly interpolated. Our search was limited to English language studies; however, the likelihood is low that the exclusion of non-English language studies would alter conclusions. ${ }^{82}$

\section{CONCLUSIONS}

We found no strong or consistent evidence that any drug class was effective in increasing abstinence, reducing use, or improving treatment retention for people with cocaine use disorder. There are several promising classes deserving of further research including psychostimulants, bupropion, topiramate, and treatment of patients with comorbid opioid use disorder.

Acknowledgments: The authors wish to thank Robin Paynter for developing the search strategy and running electronic searches.

Corresponding Author: Brian Chan, MD MPH; Division of General Internal Medicine and GeriatricsOregon Health \& Science University, 3181 SW Sam Jackson Park Road L475, Portland, OR 97239-3098, USA (e-mail: chanbri@ohsu.edu).

Funders This research was funded by the Department of Veterans Affairs, Veterans Health Administration, Office of Research and Development, Quality Enhancement Research Initiative. Dr. Chan's time was supported by grant number K12HS022981 from the Agency for Healthcare Research and Quality.

\section{Compliance with Ethical Standards:}

Conflict of Interest: The authors declare that they do not have a conflict of interest.
Disclaimer: The findings and conclusions in this document are those of the authors who are responsible for its contents; the findings and conclusions do not necessarily represent the views of the Department of Veterans Affairs or the US government.

\section{REFERENCES}

1. Lipari RN, Van Horn SL. Trends in substance use disorders among adults aged 18 or older. The CBHSQ Report, 2017. Center for Behavioral Health Statistics and Quality, Substance Abuse and Mental Health Services Administration, Rockville.

2. Goldstein RA, DesLauriers C, Burda A, Johnson-Arbor K. Cocaine: history, social implications, and toxicity: a review. Semin Diagn Pathol 2009;26(1): 10-7.

3. Riezzo I, Fiore C, De Carlo D, Pascale N, Neri M, Turillazzi E, et al. Side effects of cocaine abuse: multiorgan toxicity and pathological consequences. Curr Med Chem 2012;19(33):5624-46.

4. Pani PP, Trogu E, Vacca R, Amato L, Vecchi S, Davoli M. Disulfiram for the treatment of cocaine dependence. Cochrane Database Syst Rev 2010(1):CD007024. doi: https://doi.org/10.1002/14651858. CD007024.pub2.

5. Minozzi S, Cinquini M, Amato L, Davoli M, Farrell MF, Pani PP, et al. Anticonvulsants for cocaine dependence. Cochrane Database Syst Rev 2015(4):CD006754. doi: https://doi.org/10.1002/14651858.CD006754. pub4.

6. Minozzi S, Amato L, Pani PP, Solimini R, Vecchi S, De Crescenzo F, et al. Dopamine agonists for the treatment of cocaine dependence. Cochrane Database Syst Rev 2015(5):CD003352. doi: https://doi.org/10.1002/ 14651858.CD003352.pub4.

7. Castells X, Cunill R, Perez-Mana C, Vidal X, Capella D. Psychostimulant drugs for cocaine dependence. Cochrane Database Syst Rev 2016;9:CD007380. doi: https://doi.org/10.1002/14651858.CD007380. pub4.

8. Indave BI, Minozzi S, Pani PP, Amato L. Antipsychotic medications for cocaine dependence. Cochrane Database Syst Rev 2016;3:CD006306. doi: https://doi.org/10.1002/14651858.CD006306.pub3.

9. Moher D, Liberati A, Tetzlaff J, Altman DG, The PRISMA Group (2009). Preferred Reporting Items for Systematic Reviews and Meta-Analyses: The PRISMA Statement. PLoS Med 6(6): e1000097. doi:https://doi.org/ 10.1371/journal.pmed1000097. Available from: www.prismastatement.org.

10. Higgins J, Green S. Cochrane Handbook for Systematic Reviews of Interventions Version 5.1.0. 2011 [cited 2017 March 24]. Available from: http://handbook.cochrane.org/.

11. Kiluk BD, Carroll KM, Duhig A, Falk DE, Kampman K, Lai S, et al. Measures of outcome for stimulant trials: ACTTION recommendations and research agenda. Drug Alcohol Depend 2016;158:1-7. doi: https:// doi.org/10.1016/j.drugalcdep.2015.11.004.

12. DerSimonian R, Laird N. Meta-analysis in clinical trials. Control Clin Trials 1986;7(3): 177-88.

13. Review Manager (RevMan) [Computer program]. Version 5.3. Copenhagen: The Nordic Cochrane Centre, The Cochrane Collaboration, 2014.

14. Higgins JP, Thompson SG. Quantifying heterogeneity in a meta-analysis. Stat Med 2002;21(11):1539-58. doi: https://doi.org/10.1002/sim.1186.

15. Higgins JP, Thompson SG, Deeks JJ, Altman DG. Measuring inconsistency in meta-analyses. BMJ 2003;327(7414):557-60. doi: https://doi. org/10.1136/bmj.327.7414.557.

16. Berkman N, Lohr K, Ansari M, McDonagh M, Balk E, Whitlock E, et al. Grading the Strength of a Body of Evidence When Assessing Health Care Interventions for the Effective Health Care Program of the Agency for Healthcare Research and Quality: An Update. Rockville: Agency for Healthcare Research and Quality; Methods Guide for Comparative Effectiveness Reviews (AHRQ Publication No. 13(14)-EHC130-EF), 2013.

17. Pani PP, Trogu E, Vecchi S, Amato L. Antidepressants for cocaine dependence and problematic cocaine use. Cochrane Database Syst Rev. 2011(12):CD002950. doi: https://doi.org/10.1002/14651858. CD002950.pub3.

18. Raby WN, Rubin EA, Garawi F, Cheng W, Mason E, Sanfilippo L, et al. A randomized, double-blind, placebo-controlled trial of venlafaxine for the treatment of depressed cocaine-dependent patients. Am J Addict 2014;23(1):68-75. doi: https://doi.org/10.1111/j.1521-0391.2013.12065.x.

19. Afshar M, Knapp CM, Sarid-Segal O, Devine E, Colaneri LS, Tozier L, et al. The efficacy of mirtazapine in the treatment of cocaine dependence 
with comorbid depression. Am J Drug Alcohol Abuse 2012;38(2):181-6. doi: https://doi.org/10.3109/00952990.2011.644002.

20. Mancino MJ, McGaugh J, Chopra MP, Guise JB, Cargile C, Williams DK, et al. Clinical efficacy of sertraline alone and augmented with gabapentin in recently abstinent cocaine-dependent patients with depressive symptoms. J Clin Psychopharmacol 2014;34(2):234-9. doi: https://doi.org/ 10.1097/JCP.0000000000000062.

21. Oliveto A, Poling J, Mancino MJ, Williams DK, Thostenson J, Pruzinsky $\mathrm{R}$, et al. Sertraline delays relapse in recently abstinent cocaine-dependent patients with depressive symptoms. Addiction 2012;107(1):131-41. doi: https://doi.org/10.1111/j.1360-0443.2011.03552.x.

22. Moran LM, Phillips KA, Kowalczyk WJ, Ghitza UE, Agage DA, Epstein $\mathrm{DH}$, et al. Aripiprazole for cocaine abstinence: a randomized-controlled trial with ecological momentary assessment. Behav Pharmacol 2017;28(1):63-73. doi: https://doi.org/10.1097/FBP. 0000000000000268.

23. Poling J, Oliveto A, Petry N, Sofuoglu M, Gonsai K, Gonzalez G, et al. Sixmonth trial of bupropion with contingency management for cocaine dependence in a methadone-maintained population. Arch Gen Psychiatry 2006;63(2):219-28. doi: https://doi.org/10.1001/archpsyc.63.2.219.

24. Bisaga A, Aharonovich E, Cheng WY, Levin FR, Mariani JJ, Raby WN, et al. A placebo-controlled trial of memantine for cocaine dependence with high-value voucher incentives during a pre-randomization lead-in period. Drug Alcohol Depend 2010;111(1-2):97-104. doi: https://doi.org/10. 1016/j.drugalcdep.2010.04.006

25. Walsh SL, Middleton LS, Wong CJ, Nuzzo PA, Campbell CL, Rush CR, et al. Atomoxetine does not alter cocaine use in cocaine dependent individuals: double blind randomized trial. Drug Alcohol Depend 2013;130(1-3):150-7. doi: https://doi.org/10.1016/j.drugalcdep.2012. 10.024 .

26. Winhusen TM, Kropp F, Lindblad R, Douaihy A, Haynes L, Hodgkins C, et al. Multisite, randomized, double-blind, placebo-controlled pilot clinical trial to evaluate the efficacy of buspirone as a relapse-prevention treatment for cocaine dependence. J Clin Psychiatry 2014;75(7):757-64. doi: https://doi.org/10.4088/JCP.13m08862.

27. Shoptaw S, Yang X, Rotheram-Fuller EJ, Hsieh Y-CM, Kintaudi PC, Charuvastra VC, et al. Randomized placebo-controlled trial of baclofen for cocaine dependence: preliminary effects for individuals with chronic patterns of cocaine use. J Clin Psychiatry 2003;64(12):1440-8.

28. Kahn R, Biswas K, Childress A-R, Shoptaw S, Fudala PJ, Gorgon L, et al. Multi-center trial of baclofen for abstinence initiation in severe cocainedependent individuals. Drug Alcohol Depend 2009;103(1-2):59-64. doi: https://doi.org/10.1016/j.drugalcdep.2009.03.011.

29. Baldacara L, Cogo-Moreira H, Parreira BL, Diniz TA, Milhomem JJ, Fernandes CC, et al. Efficacy of topiramate in the treatment of crack cocaine dependence: a double-blind, randomized, placebo-controlled trial. J Clin Psychiatry 2016;77(3):398-406. doi: https://doi.org/10. 4088/JCP. 14m09377

30. Johnson BA, Ait-Daoud N, Wang X-Q, Penberthy JK, Javors MA, Seneviratne C, et al. Topiramate for the treatment of cocaine addiction: a randomized clinical trial. JAMA Psychiatry 2013;70(12):1338-46. doi: https://doi.org/10.1001/jamapsychiatry.2013.2295

31. Kampman KM, Pettinati H, Lynch KG, Dackis C, Sparkman T, Weigley C, et al. A pilot trial of topiramate for the treatment of cocaine dependence. Drug Alcohol Depend 2004;75(3):233-40. doi: https://doi.org/10.1016/ j.drugalcdep.2004.03.008

32. Kampman KM, Pettinati HM, Lynch KG, Spratt K, Wierzbicki MR, O'Brien CP. A double-blind, placebo-controlled trial of topiramate for the treatment of comorbid cocaine and alcohol dependence. Drug Alcohol Depend 2013;133(1):94-9. doi: https://doi.org/10.1016/j.drugalcdep.2013.05. 026

33. Nuijten M, Blanken P, van den Brink W, Hendriks V. Treatment of crackcocaine dependence with topiramate: a randomized controlled feasibility trial in The Netherlands. Drug Alcohol Depend 2014;138:177-84. doi: https://doi.org/10.1016/j.drugalcdep.2014.02.024

34. Umbricht A, DeFulio A, Winstanley EL, Tompkins DA, Peirce J, Mintzer MZ, et al. Topiramate for cocaine dependence during methadone maintenance treatment: a randomized controlled trial. Drug Alcohol Depend 2014;140:92-100. doi: https://doi.org/10.1016/j.drugalcdep. 2014.03.033

35. Schottenfeld RS, Pakes JR, Oliveto A, Ziedonis D, Kosten TR Buprenorphine vs methadone maintenance treatment for concurrent opioid dependence and cocaine abuse. Arch Gen Psychiatry 1997;54(8):713-20.

36. Schottenfeld RS, Chawarski MC, Pakes JR, Pantalon MV, Carroll KM, Kosten TR. Methadone versus buprenorphine with contingency management or performance feedback for cocaine and opioid dependence. Am J Psychiatry 2005;162(2):340-9. doi: https://doi.org/10. 1176/appi.ajp.162.2.340

37. Carroll KM, Nich C, Petry NM, Eagan DA, Shi JM, Ball SA. A randomized factorial trial of disulfiram and contingency management to enhance cognitive behavioral therapy for cocaine dependence. Drug Alcohol Depend 2016;160:135-42. doi: https://doi.org/10.1016/j.drugalcdep. 2015.12.036

38. Carroll KM, Nich C, Shi JM, Eagan D, Ball SA. Efficacy of disulfiram and Twelve Step Facilitation in cocaine-dependent individuals maintained on methadone: a randomized placebo-controlled trial. Drug Alcohol Depend 2012;126(1-2):224-31. doi: https://doi.org/10.1016/j.drugalcdep.2012. 05.019

39. Kosten TR, Wu G, Huang W, Harding MJ, Hamon SC, Lappalainen J, et al. Pharmacogenetic randomized trial for cocaine abuse: disulfiram and dopamine beta-hydroxylase. Biol Psychiatry 2013;73(3):219-24. doi: https://doi.org/10.1016/j.biopsych.2012.07.011

40. Oliveto A, Poling J, Mancino MJ, Feldman Z, Cubells JF, Pruzinsky R, et al. Randomized, double blind, placebo-controlled trial of disulfiram for the treatment of cocaine dependence in methadone-stabilized patients. Drug Alcohol Depend 2011;113(2-3):184-91. doi: https://doi.org/10. 1016/j.drugalcdep.2010.07.022

41. Schottenfeld RS, Chawarski MC, Cubells JF, George TP, Lappalainen J, Kosten TR. Randomized clinical trial of disulfiram for cocaine dependence or abuse during buprenorphine treatment. Drug Alcohol Depend 2014;136:36-42. doi: https://doi.org/10.1016/j.drugalcdep.2013.12. 007 .

42. Shorter D, Nielsen DA, Huang W, Harding MJ, Hamon SC, Kosten TR. Pharmacogenetic randomized trial for cocaine abuse: disulfiram and alphalA-adrenoceptor gene variation. Eur Neuropsychopharmacol 2013;23(11):1401-7. doi: https://doi.org/10.1016/j.euroneuro.2013.05. 014

43. Pettinati HM, Kampman KM, Lynch KG, Dundon WD, Mahoney EM, Wierzbicki MR, et al. A pilot trial of injectable, extended-release naltrexone for the treatment of co-occurring cocaine and alcohol dependence. Am J Addict 2014;23(6):591-7. doi: https://doi.org/10.1111/j.15210391.2014.12146.x

44. Schmitz JM, Lindsay JA, Green CE, Herin DV, Stotts AL, Moeller FG. High-dose naltrexone therapy for cocaine-alcohol dependence. Am J Addict 2009;18(5):356-62. doi: https://doi.org/10.3109/ 10550490903077929

45. Schmitz JM, Stotts AL, Sayre SL, DeLaune KA, Grabowski J. Treatment of cocaine-alcohol dependence with naltrexone and relapse prevention therapy. Am J Addict 2004;13(4):333-41. doi: https://doi.org/10.1080/ 10550490490480982

46. Schmitz JM, Stotts AL, Rhoades HM, Grabowski J. Naltrexone and relapse prevention treatment for cocaine-dependent patients. Addict Behav 2001:26(2):167-80.

47. Hersh D, Van Kirk JR, Kranzler HR. Naltrexone treatment of comorbid alcohol and cocaine use disorders. Psychopharmacology. 1998;139(12):44-52.

48. Pettinati HM, Kampman KM, Lynch KG, Suh JJ, Dackis CA, Oslin DW, et al. Gender differences with high-dose naltrexone in patients with cooccurring cocaine and alcohol dependence. J Subst Abus Treat 2008;34(4):378-90. doi: https://doi.org/10.1016/j.jsat.2007.05.011

49. Ling W, Hillhouse MP, Saxon AJ, Mooney LJ, Thomas CM, Ang A, et al. Buprenorphine + naloxone plus naltrexone for the treatment of cocaine dependence: the Cocaine Use Reduction with Buprenorphine (CURB) study. Addiction 2016;111(8):1416-27. doi: https://doi.org/10.1111/ add. 13375

50. Kampman KM, Dackis C, Pettinati HM, Lynch KG, Sparkman T, O’Brien CP. A double-blind, placebo-controlled pilot trial of acamprosate for the treatment of cocaine dependence. Addict Behav 2011;36(3):217-21. doi: https://doi.org/10.1016/j.addbeh.2010.11.003

51. Poling J, Rounsaville B, Gonsai K, Severino K, Sofuoglu M. The safety and efficacy of varenicline in cocaine using smokers maintained on methadone: a pilot study. Am J Addict 2010;19(5):401-8. doi: https://doi.org/ 10.1111/j.1521-0391.2010.00066.x

52. Plebani JG, Lynch KG, Yu Q, Pettinati HM, O’Brien CP, Kampman KM. Results of an initial clinical trial of varenicline for the treatment of cocaine dependence. Drug Alcohol Depend 2012;121(1-2):163-6. doi: https:// doi.org/10.1016/j.drugalcdep.2011.08.025

53. Malcolm R, LaRowe S, Cochran K, Moak D, Herron J, Brady K, et al. A controlled trial of amlodipine for cocaine dependence: a negative report. J Subst Abus Treat 2005;28(2):197-204. doi: https://doi.org/10.1016/j. jsat.2004.12.006 
54. Sofuoglu M, Poling J, Babuscio T, Gonsai K, Severino K, Nich C, et al. Carvedilol does not reduce cocaine use in methadone-maintained cocaine users. J Subst Abus Treat 2017;73:63-9. doi: https://doi.org/10.1016/j. jsat.2016.11.005

55. Reid MS, Angrist B, Baker S, Woo C, Schwartz M, Montgomery A, et al. A placebo-controlled screening trial of celecoxib for the treatment of cocaine dependence. Addiction 2005;100 Suppl 1:32-42. doi: https://doi.org/10. 1111/j.1360-0443.2005.00989.x.

56. Licata SC, Penetar DM, Ravichandran C, Rodolico J, Palmer C, Berko J, et al. Effects of daily treatment with citicoline: a double-blind, placebocontrolled study in cocaine-dependent volunteers. J Addict Med 2011;5(1):57-64. doi: https://doi.org/10.1097/ADM. Ob013e3181d80c93

57. Kennedy AP, Gross RE, Whitfield N, Drexler KPG, Kilts CD. A controlled trial of the adjunct use of D-cycloserine to facilitate cognitive behavioral therapy outcomes in a cocaine-dependent population. Addict Behav 2012;37(8):900-7. doi: https://doi.org/10.1016/j.addbeh.2012.03.008

58. Shoptaw S, Majewska MD, Wilkins J, Twitchell G, Yang X, Ling W. Participants receiving dehydroepiandrosterone during treatment for cocaine dependence show high rates of cocaine use in a placebocontrolled pilot study. Exp Clin Psychopharmacol 2004;12(2):126-35 doi: https://doi.org/10.1037/1064-1297.12.2.126

59. Shorter D, Lindsay JA, Kosten TR. The alpha-1 adrenergic antagonist doxazosin for treatment of cocaine dependence: A pilot study. Drug Alcohol Depend 2013;131(1-2):66-70. doi: https://doi.org/10.1016/j. drugalcdep.2012.11.021

60. Sofuoglu M, Carroll KM. Effects of galantamine on cocaine use in chronic cocaine users. Am J Addict 2011;20(3):302-3. doi: https://doi.org/10 1111/j.1521-0391.2011.00130.x

61. Margolin A, Kantak K, Copenhaver M, Avants SK. A preliminary, controlled investigation of magnesium L-aspartate hydrochloride for illicit cocaine and opiate use in methadone-maintained patients. J Addict Dis 2003;22(2):49-61. doi: https://doi.org/10.1300/J069v22n02_04

62. Reid MS, Angrist B, Baker SA, O'Leary S, Stone J, Schwartz M, et al. A placebo controlled, double-blind study of mecamylamine treatment for cocaine dependence in patients enrolled in an opiate replacement program. Subst Abus 2005;26(2):5-14.

63. Kablinger AS, Lindner MA, Casso S, Hefti F, DeMuth G, Fox BS, et al. Effects of the combination of metyrapone and oxazepam on cocaine craving and cocaine taking: a double-blind, randomized, placebocontrolled pilot study. J Psychopharmacol 2012;26(7):973-81. doi: https://doi.org/10.1177/0269881111430745

64. Mariani JJ, Pavlicova M, Bisaga A, Nunes EV, Brooks DJ, Levin FR. Extended-release mixed amphetamine salts and topiramate for cocaine dependence: a randomized controlled trial. Biol Psychiatry 2012;72(11):950-6. doi: https://doi.org/10.1016/j.biopsych.2012.05. 032

65. LaRowe SD, Kalivas PW, Nicholas JS, Randall PK, Mardikian PN, Malcolm RJ. A double-blind placebo-controlled trial of N-acetylcysteine in the treatment of cocaine dependence. Am J Addict 2013;22(5):443-52. doi: https://doi.org/10.1111/j.1521-0391.2013.12034.x

66. Johnson BA, Roache JD, Ait-Daoud N, Javors MA, Harrison JM, Elkashef A, et al. A preliminary randomized, double-blind, placebo-controlled study of the safety and efficacy of ondansetron in the treatment of cocaine dependence. Drug Alcohol Depend 2006;84(3):256-63. doi: https://doi.org/10.1016/j.drugalcdep.2006.02.011

67. Schmitz JM, Green CE, Hasan KM, Vincent J, Suchting R, Weaver MF, et al. PPAR-gamma agonist pioglitazone modifies craving intensity and brain white matter integrity in patients with primary cocaine use disorder: a double-blind randomized controlled pilot trial. Addiction 2017;112(10):1861-8. doi: https://doi.org/10.1111/add.13868

68. Kampman K, Majewska MD, Tourian K, Dackis C, Cornish J, Poole S, et al. A pilot trial of piracetam and ginkgo biloba for the treatment of cocaine dependence. Addict Behav 2003;28(3):437-48.

69. Sofuoglu M, Poling J, Gonzalez G, Gonsai K, Oliveto A, Kosten TR. Progesterone effects on cocaine use in male cocaine users maintained on methadone: a randomized, double-blind, pilot study. Exp Clin Psychopharmacol 2007;15(5):453-60. doi: https://doi.org/10.1037/ 1064-1297.15.5.453
70. Kampman KM, Volpicelli JR, Mulvaney F, Alterman AI, Cornish J, Gariti $\mathrm{P}$, et al. Effectiveness of propranolol for cocaine dependence treatment may depend on cocaine withdrawal symptom severity. Drug Alcohol Depend 2001;63(1):69-78.

71. Gilgun-Sherki Y, Eliaz RE, McCann DJ, Loupe PS, Eyal E, Blatt K, et al. Placebo-controlled evaluation of a bioengineered, cocaine-metabolizing fusion protein, TV-1380 (AlbuBChE), in the treatment of cocaine dependence. Drug Alcohol Depend 2016;166:13-20. doi: https://doi. org/10.1016/j.drugalcdep.2016.05.019

72. Castells X, Kosten TR, Capella D, Vidal X, Colom J, Casas M. Efficacy of opiate maintenance therapy and adjunctive interventions for opioid dependence with comorbid cocaine use disorders: A systematic review and meta-analysis of controlled clinical trials. Am J Drug Alcohol Abuse 2009;35(5):339-49. doi: https://doi.org/10.1080/00952990903108215

73. Ahmad FB, Rossen LM, Spencer MR, Warner M, Sutton P. Provisional drug overdose death counts. National Center for Health Statistics. 2018. Designed by LM Rossen, A Lipphardt, FB Ahmad, JM Keralis, and Y Chong: National Center for Health Statistics. Available from https://www. cdc.gov/nchs/nvss/vsrr/drug-overdose-data.htm.

74. Singh M, Keer D, Klimas J, Wood E, Werb D. Topiramate for cocaine dependence: a systematic review and meta-analysis of randomized controlled trials. Addiction 2016;111(8):1337-46. doi: https://doi.org/ 10.1111/add. 13328

75. McCall Jones C, Baldwin GT, Compton WM. Recent Increases in CocaineRelated Overdose Deaths and the Role of Opioids. Am J Public Health 2017;107(3):430-2. doi: https://doi.org/10.2105/ajph.2016.303627.

76. Leeman RF, Sun Q, Bogart D, Beseler CL, Sofuoglu M. Comparisons of Cocaine-Only, Opioid-Only, and Users of Both Substances in the National Epidemiologic Survey on Alcohol and Related Conditions (NESARC). Subst Use Misuse 2016;51(5):553-64. doi: https://doi.org/10.3109/ 10826084.2015.1122063.

77. Phillips KA, Epstein DH, Preston KL. Psychostimulant addiction treatment. Neuropharmacology. 2014;87:150-60. https://doi.org/10.1016/j. neuropharm.2014.04.002.

78. Minozzi S, Saulle R, De Crescenzo F, Amato L. Psychosocial interventions for psychostimulant misuse. Cochrane Database Syst Rev 2016;9:CD011866. doi: https://doi.org/10.1002/14651858.CD011866.pub2.

79. Klimas J, Wood E, Werb D. How can we investigate the role of topiramate in the treatment of cocaine use disorder more thoroughly? Addiction 2017;112(1):182-3. doi: https://doi.org/10.1111/add.13618.

80. Darke S, Farrell M. Commentary on Singh et al. (2016): Still searching for the answer. Addiction 2016;111(8):1347. doi: https://doi.org/10.1111/ add. 13369 .

81. Chan B, Kondo K, Ayers C, Freeman M, Montgomery J, Paynter R, et al. Pharmacotherapy for Stimulant Use Disorders: A Systematic Review of the Evidence. VA ESP Project 05-225; 2018.

82. Moher D, Pham B, Lawson ML, Klassen TP. The inclusion of reports of randomised trials published in languages other than English in systematic reviews. Health Technol Assess 2003;7(41):1-90

83. Brodie JD, Case BG, Figueroa E, Dewey SL, Robinson JA, Wanderling JA et al. Randomized, double-blind, placebo-controlled trial of vigabatrin for the treatment of cocaine dependence in Mexican parolees. Am J Psychiatry 2009;166(11):1269-77. doi: https://doi.org/10.1176/appi. ajp.2009.08121811

84. Somoza EC, Winship D, Gorodetzky CW, Lewis D, Ciraulo DA, Galloway GP, et al. A multisite, double-blind, placebo-controlled clinical trial to evaluate the safety and efficacy of vigabatrin for treating cocaine dependence. JAMA Psychiatry 2013;70(6):630-7. doi: https://doi.org/ 10.1001/jamapsychiatry.2013.872

85. George TP, Chawarski MC, Pakes J, Carroll KM, Kosten TR, Schottenfeld RS. Disulfiram versus placebo for cocaine dependence in buprenorphinemaintained subjects: a preliminary trial. Biol Psychiatry 2000;47(12): 1080-6.

86. Petrakis IL, Carroll KM, Nich C, Gordon LT, McCance-Katz EF, Frankforter T, et al. Disulfiram treatment for cocaine dependence in methadone-maintained opioid addicts. Addiction 2000;95(2):219-28.

Publisher's Note Springer Nature remains neutral with regard to jurisdictional claims in published maps and institutional affiliations. 